\title{
Efficacy of Acupuncture-Related Therapy in the Treatment of Knee Osteoarthritis: A Network Meta-Analysis of Randomized Controlled Trials
}

\author{
Wei Liu ${ }^{1,2, *}$ \\ Yihua Fan (D) ${ }^{1,2, *}$ \\ Yuanhao $\mathrm{Wu} \mathbb{( D}^{1,2, *}$ \\ $\mathrm{Xu} \mathrm{Hou}{ }^{3}$ \\ Bin Xue ${ }^{1,2}$ \\ Peihao Li (D) ${ }^{1,2}$ \\ Shumin Zhang $\mathbb{D}^{1,2}$ \\ Qingyun Yue $\mathbb{D}^{1,2}$
}

'First Teaching Hospital of Tianjin University of Traditional Chinese Medicine, Tianjin, 300193, People's Republic of China; ${ }^{2}$ National Clinical Research Center for Chinese Medicine Acupuncture and Moxibustion, Tianjin, 30038I, People's Republic of China;

${ }^{3}$ Department of Endocrinology and Metabolic Diseases, Shandong Provincial Hospital Affiliated to Shandong First Medical University, Jinan, Shandong Province, 25002I, People's Republic of China

*These authors contributed equally to this work
Correspondence: Wei Liu No. 88, Chang Ling Road, Li Qi Zhuang Jie, Xi Qing District, Tianjin, People's Republic of China

Email fengshiliuwei@।63.com
Objective: Knee osteoarthritis (KOA) is prevalent in middle-aged and elderly people. This condition negatively affects the quality of life of patients. Although non-steroidal antiinflammatory drugs (NSAIDs) are often used to relieve symptoms associated with KOA, it is associated with many side effects. Acupuncture and moxibustion therapies have been applied in the treatment of KOA. However, the efficacy of various acupuncture and moxibustion treatments has not been compared.

Methods: Randomized controlled trials (RCTs) on the application of acupuncture and moxibustion in the treatment of KOA were searched in English databases and Chinese databases. Data were retrieved from establishment of the database to September 2020. Data analysis was performed using Stata14.0 and GeMTC 0.14.3 softwares.

Results: A total of 40 RCTs involving 3215 patients with KOA were retrieved. Network meta-analysis revealed that the fire needle was superior to western medicine, electro-acupuncture, conventional acupuncture, warm needle and sham acupuncture; warm needle was better than conventional acupuncture and western medicine whereas electro-acupuncture was better than conventional acupuncture in improving pain scores in the Western Ontario and McMaster Universities Osteoarthritis Index (WOMAC). Moreover, we found that fire needle and warm needle more effectively improved WOMAC stiffness scores than western medicine and sham moxibustion, whereas electro-acupuncture was superior to western medicine and sham moxibustion in improving WOMAC stiffness scores. Further analysis revealed that fire needle, warm needle and electro-acupuncture were more effective in improving WOMAC joint function scores than conventional acupuncture and western medicine. The fire needle was superior to conventional acupuncture and sham acupuncture, whereas electroacupuncture was better than western medicine, conventional acupuncture and sham acupuncture in improving visual analogue scale scores.

Conclusion: This study shows that fire needle is superior to warm needle and electroacupuncture, whereas warm needle and electro-acupuncture are better than conventional acupuncture, western medicine, sham moxibustion and sham acupuncture.

Keywords: knee osteoarthritis, acupuncture, moxibustion, randomized controlled trials, network meta-analysis

\section{Introduction}

Knee osteoarthritis (KOA) is a common disease associated with knee joint degeneration among the elderly. The disease has seriously negative effects on the quality of life of patients. Moreover, it is one of the main diseases leading to knee dysfunction and disability among the elderly people. ${ }^{1,2}$ Globally, KOA is the 11 th 
leading cause of disability, affecting about $3.8 \%$ of the world's population. ${ }^{3}$ With the progressively increasing aging population in China, the incidence of KOA has been on the rise, reaching about $85 \%$ among those aged over 65 years. ${ }^{4}$ The development of KOA is associated with a variety of factors, including age, sex, aging, trauma, obesity, inflammation, occupation, activity, metabolism, and heredity among others. ${ }^{5}$ Non-steroidal anti-inflammatory drugs (NSAIDs) are recommended for the treatment of early and middle-term KOA. ${ }^{6}$ Although their analgesic effects are very good, patients often develop pains after drug withdrawal. Cases of gastrointestinal discomfort, liver and kidney function damage as well as other adverse reactions have also been reported. ${ }^{7}$ Acupuncture and moxibustion have shown good therapeutic effects on KOA with few adverse reactions, ${ }^{8,9}$ and have been adopted in China's Guidelines for the Diagnosis and Treatment of Osteoarthritis $^{7}$ and the Guidelines of the American Academy of Orthopaedic Surgeons. ${ }^{10}$ There are many types of acupuncture and moxibustion treatments, with varying clinical effects. Direct comparisons of the curative effects of different acupuncture and moxibustion therapies have not been done so far. Therefore, we used network meta-analysis to compare the efficacy of different types of acupuncture and moxibustion therapies in KOA patients to provide a basis for selection of optimal acupuncture and moxibustion therapies in the clinical treatment of KOA.

\section{Information and Methodology Research Registration}

The network meta-analysis research protocol was registered on the PROSPERO at https://www.crd.york.ac.uk/ prospero/\#recordDetails; Registration number: CRD42020203602. This network meta-analysis was conducted according to the Preferred Reporting Items for Systematic Reviews and Meta-Analyses for NMA guidelines. See supplementary materials (Table S1).

\section{Inclusion and Exclusion Criteria}

i. Study type: randomized controlled trials (RCTs), not limited to blinding method, but limited to Chinese and English languages.

ii. Study participants: KOA patients diagnosed based on definitive diagnostic criteria, gender and age were unlimited.

iii. Interventions: treatment groups involved different acupuncture therapies, including conventional acupuncture alone, warm needle, electro-acupuncture, fire needle, blood-letting puncture, moxibustion, auricular acupuncture, auricular point sticking, acupoint catgut embedding and acupoint injection, control groups comprised of treatments such as western medicine, sham acupuncture and sham moxibustion; or a comparison between different acupuncture types.

iv. Outcome indicators: a. pain, stiffness, and joint function scores based on the Western Ontario and McMaster University Osteoarthritis Index (WOMAC); b. Visual Analogue Scale (VAS); c. Adverse events.

v. Exclusion criteria: a. Studies in which participants did not conform to the inclusion criteria, such as patients with other arthritis; $b$. studies without clear diagnostic criteria; c. Studies without any of the above outcome indicators; $d$. Studies using acupuncture combination therapy, such as acupuncture combined with moxibustion, acupuncture combined with auricular acupuncture treatment; e. Studies using traditional Chinese medicine treatments in both groups, such as cupping and Chinese medicine compounds; f. For repeated publications, studies with the most complete data were selected; g. Abstracts or articles without specific data on relevant indicators, and which could not be obtained from the corresponding authors.

\section{Search Strategy}

Published RCTs on the application of acupuncture and moxibustion in the treatment of KOA were searched in PubMed, Cochrane Library, EMBASE, Web of Science, CNKI, VIP, Wanfang and China Biomedical Literature Databases. Chinese search terms were "zhen ci" (acupuncture), "dian zhen" (electro-acupuncture), "wen zhen jiu" (warm needle), "huo zhen" (fire needle), "ci luo" (bloodletting puncture), "ai jiu" (moxibustion), "er xue tie ya" (auricular acupoint sticking), "er zhen" (auricular acupuncture), "xue wei mai xian" (acupoint catgut embedding), "xue wei zhu she" (acupoint injection), "xi gu guan jie yan" (knee osteoarthritis). English search terms were "acupuncture", "electro-acupuncture ", "warm needle", "fire needle", "blood-letting puncture", "moxibustion", "auricular application pressure", "auricular needle", "acupoint catgut embedding", "acupoint injection", "knee osteoarthritis", "KOA". PubMed database retrieval strategies are shown in Table 1.

\section{Studies Screening and Data Extraction}

Study screening and data extraction, as well as crosschecking, were independently performed by two 
Table I Retrieval Strategy of Studies from the PubMed Database

\begin{tabular}{|c|c|}
\hline Number & Search Terms \\
\hline$\# 1$ & Acupuncture $[\mathrm{MeSH}]$ \\
\hline \#2 & Acupuncture [Title/Abstract] \\
\hline \#3 & Pharmacopuncture [Title/Abstract] \\
\hline$\# 4$ & Electro-acupuncture [Title/Abstract] \\
\hline$\# 5$ & Warm needle [Title/Abstract] \\
\hline \#6 & Fire needle [Title/Abstract] \\
\hline \#7 & Blood-letting puncture [Title/Abstract] \\
\hline \#8 & Moxibustion $[\mathrm{MeSH}]$ \\
\hline \#9 & Moxibustion [Title/Abstract] \\
\hline \#10 & Auricular application pressure [Title/Abstract] \\
\hline \#II & Auricular needle [Title/Abstract $]$ \\
\hline$\# 12$ & Acupoint catgut embedding [Title/Abstract] \\
\hline \#13 & Acupoint injection [Title/Abstract] \\
\hline \#14 & \#IOR\#2OR\#3OR\#4OR\#5OR\#6OR\#7OR\#8OR\#9OR\#I0OR\#IIOR\#I2OR\#I3 \\
\hline \#15 & Osteoarthritis, knee $[\mathrm{MeSH}]$ \\
\hline \#16 & Osteoarthritis, knee [Title/Abstract] \\
\hline \#17 & Knee osteoarthritis [Title/Abstract] \\
\hline \#18 & Knee osteoarthritides [Title/Abstract] \\
\hline \#19 & Osteoarthritis of knee [Title/Abstract] \\
\hline \#20 & Osteoarthritis of the knee [Title/Abstract] \\
\hline \#21 & KOA [Title/Abstract] \\
\hline \#22 & \#I5OR\#I6OR\#I7OR\#I8OR\#I9OR\#200R\#2I \\
\hline \#23 & \#I4AND\#22 \\
\hline
\end{tabular}

researchers. In case of disagreements, a third researcher was involved to reach a consensus. The following information was obtained: name of first author, publication year, KOA diagnostic criteria, sample size, gender, age, course of disease, study type, intervention, treatment course, and outcome indicators.

\section{Risk Assessment of Bias in the Included Studies}

The Cochrane System Evaluation Manual version 5.1.0 RCT bias risk assessment tool was used to evaluate the quality of the included studies. This was done through random sequence generation, allocation concealment, participant and personnel blinding, outcome assessment blinding, incomplete outcome data, selective reporting, and other bias items. Two researchers graded the above contents as "low risk", "high risk" and "unclear", and cross-checked the obtained results. A third researcher was consulted if there were any disagreements. Finally, a bias risk diagram was drawn using RevMan5.3 software.

\section{Statistical Analysis}

Stata 14.0 software was used to draw an evidence network diagram to show comparisons of the intervention measures for each outcome indicator. For continuous variables, if the unit or tool of the measurement index was the same, the mean difference (MD) was used for analysis; if the measurement tools or units were inconsistent, the standardized mean difference (SMD) was used for analysis. Chi-square test was used to directly compare heterogeneity between research results, and $I^{2}$ was used to determine level of heterogeneity. If results of the included studies showed no statistical heterogeneity $\left(I^{2}<50 \%, P>0.1\right)$, a metaanalysis using the fixed effect model. If heterogeneity was found, the reasons for heterogeneity were further analyzed. If there was no obvious clinical heterogeneity or methodological heterogeneity, the random effect model was used for meta-analysis. Small sample effects or publication bias were detected using comparative corrected funnel plots. The GEMTC 0.14.3 software was used for network metaanalysis based on the Markov Chain Monte Carlo (MCMC) fitting consistent model under the Bayesian framework. Four chains were used for simulation, and the number of iterations was set at 50,000. The potential scale reduction factor (PSRF) was estimated and deduced under the assumption that MCMC reached a stable convergence state. The stability and consistency of results were evaluated using the MCMC fitted inconsistency model. 


\section{Results}

\section{Study Retrieval Results}

A total of 6290 relevant studies were retrieved. After primary screening and re-screening, $40 \mathrm{RCTs}^{11-50}$ involving 3215 patients were finally included in the study. The screening process of the included studies is shown in Figure 1.

\section{Basic Features of the Included Studies} Among the 40 RCTs, ${ }^{11-50} 6,17,16,12,9,1,18,1,1$ and 1 RCTs involved the application of moxibustion, electroacupuncture, warm acupuncture, conventional acupuncture, fire needle, acupoint embedding, western medicine, placebo, sham acupuncture, and sham moxibustion, respectively. Among these studies, there were 2 threearm trials ${ }^{12,38}$ and 38 double-arm trials; ${ }^{11,13-37,39-50}$ In addition, 23 trials $^{13,14,18,20-22,24-28,31-34,36,40-43,46,49,50}$ reported WOMAC pain scores, $21^{13,14,18,20-22,24-28,31-}$
34,36,37,42,43,46,49 reported WOMAC stiffness scores, 2113,14,18,20-22,25-28,31-34,36,37,40-42,46,49 reported WOMAC joint function scores, 25 $5^{11-13,15-17,19,22,23,25,29,30,34-}$ 36,38,40,42-49 reported VAS scores while 12$11,20,24,31,32,37,39-41,44,48,49$ reported adverse events. Basic characteristics of the included studies are shown in Table 2, whereas the characteristics of the interventions are shown in Table 3 .

\section{Risk of Bias Assessment Results of the Included Studies}

i. Random sequence generation: Nineteen studies$12,14,18,20,22,25,26,28,29,32,36,37,39,42-44,46,48,49$ used a table of random numbers, seven ${ }^{13,16,21,35,40,41,50}$ used computer-generated random numbers, one ${ }^{17}$ used a coin toss for randomization, one $^{19}$ used random cards, while the remaining twelve$11,15,23,24,27,30,31,33,34,38,45,47$ only mentioned the word "random"; ii. Allocation concealment: Three studies ${ }^{13,28,36}$ used
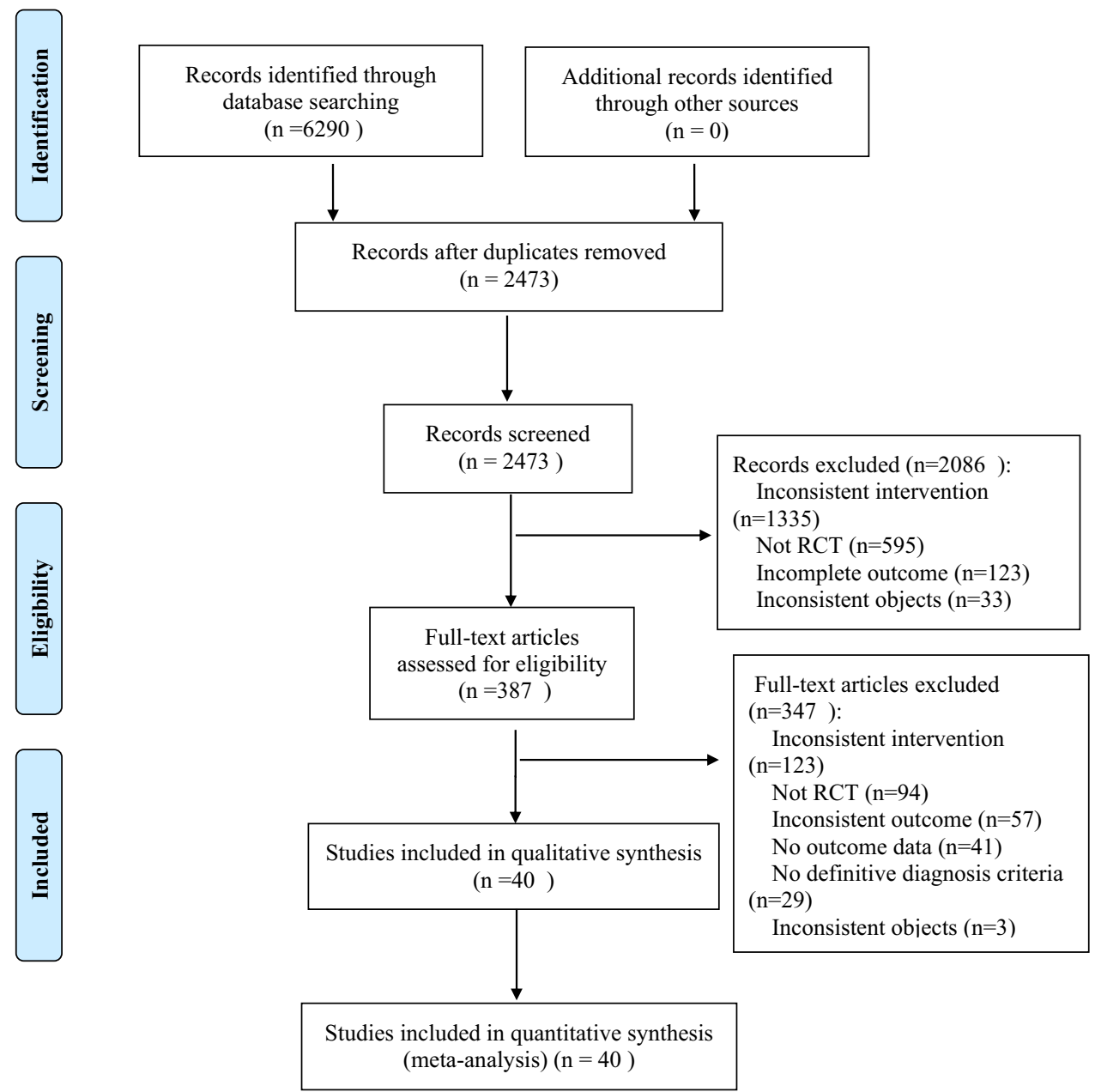

Figure I Flowchart of study screening. 
Table 2 Basic Features of the Included Studies

\begin{tabular}{|c|c|c|c|c|c|}
\hline Included Studies & Diagnostic Criteria & $\begin{array}{l}\text { Sample } \\
\text { Size (T/ } \\
\text { C) }\end{array}$ & $\begin{array}{l}\text { Sex } \\
\text { (Men/ } \\
\text { Women) }\end{array}$ & Age (Year) & $\begin{array}{l}\text { Course of Disease } \\
\text { (Year) }\end{array}$ \\
\hline Zhang $2011^{11}$ & $A C R$ & $30 / 30$ & $22 / 38$ & 58.2 & - \\
\hline Zhou $2014^{12}$ & $A C R$ & $39 / 44 / 22$ & $\begin{array}{l}\mathrm{T}: 14 / 25 \\
\mathrm{C} 1: 8 / 36 \\
\mathrm{C} 2: 5 / 17\end{array}$ & $\begin{array}{l}\mathrm{T}: 67 \pm 10 \\
\mathrm{Cl}: 80 \pm 10 \\
\mathrm{C} 2: 66 \pm 12\end{array}$ & $\begin{array}{l}\mathrm{T}: 3 \\
\mathrm{Cl}: 2.02 \\
\mathrm{C} 2: 1.53\end{array}$ \\
\hline Zhou $2017^{13}$ & $A C R$ & $30 / 30$ & $\begin{array}{l}T: 14 / 16 \\
C: 13 / 17\end{array}$ & $\begin{array}{l}\mathrm{T}: 59.07 \pm 7.89 \\
\mathrm{C}: 60.60 \pm 8.27\end{array}$ & $\begin{array}{l}\mathrm{T}: 5.73 \pm 2.85 \\
\mathrm{C}: 5.50 \pm 2.54\end{array}$ \\
\hline Ren $2011^{14}$ & $A C R$ & $31 / 28$ & $\begin{array}{l}\mathrm{T}: 1 \mathrm{I} / 20 \\
\mathrm{C}: 8 / 20\end{array}$ & $\begin{array}{l}\mathrm{T}: 64.03 \pm 7.24 \\
\mathrm{C}: 62.57 \pm 8.12\end{array}$ & $\begin{array}{l}\mathrm{T}: 6.82 \pm 6.60 \\
\mathrm{C}: 7.15 \pm 7.72\end{array}$ \\
\hline Zhou $2015^{15}$ & ACR & $40 / 40$ & $\begin{array}{l}T: 17 / 23 \\
C: 19 / 21\end{array}$ & $\begin{array}{l}\mathrm{T}: 54.6 \pm 5.3 \\
\mathrm{C}: 53.8 \pm 7.6\end{array}$ & $\begin{array}{l}\mathrm{T}: 17.2 \pm 2.2 \text { (month) } \\
\mathrm{C}: 15.6 \pm 3.0 \text { (month) }\end{array}$ \\
\hline Zhang $2018^{16}$ & ACR & $39 / 39$ & $\begin{array}{l}T: 13 / 26 \\
C: 15 / 24\end{array}$ & $\begin{array}{l}\mathrm{T}: 61.36 \pm 2.24 \\
\mathrm{C}: 62.08 \pm 2.46\end{array}$ & $\begin{array}{l}\mathrm{T}: 4.77 \pm 0.12 \\
\mathrm{C}: 4.68 \pm 0.28\end{array}$ \\
\hline Liu $2020^{17}$ & $\begin{array}{l}\text { Guidelines for the diagnosis and treatment of } \\
\text { osteoarthritis }\end{array}$ & $41 / 37$ & $\begin{array}{l}\mathrm{T}: 2 / 39 \\
\mathrm{C}: 3 / 34\end{array}$ & $\begin{array}{l}\mathrm{T}: 61.72 \pm 8.05 \\
\mathrm{C}: 60.98 \pm 7.56\end{array}$ & $\begin{array}{l}\mathrm{T}: 2.13 \pm 0.98 \\
\mathrm{C}: 2.53 \pm 0.83\end{array}$ \\
\hline Guo $2016^{18}$ & $\begin{array}{l}\text { Guidelines for the diagnosis and treatment of } \\
\text { osteoarthritis }\end{array}$ & $45 / 45$ & $\begin{array}{l}T: 19 / 26 \\
C: 17 / 28\end{array}$ & $\begin{array}{l}T: 56.00 \pm 7.25 \\
C: 57.17 \pm 6.96\end{array}$ & $\begin{array}{l}\mathrm{T}: 33.75 \pm 14.1 \mathrm{I} \text { (month) } \\
\mathrm{C}: 32.35 \pm 13.72 \text { (month) }\end{array}$ \\
\hline Wu $2015^{19}$ & $A C R$ & $47 / 48$ & - & $\begin{array}{l}\mathrm{T}: 58.75 \pm \mathrm{I} .2 \mathrm{I} \\
\mathrm{C}: 60.02 \pm 2.17\end{array}$ & $\begin{array}{l}T: 18 \pm 2 \text { (month) } \\
C: 19 \pm 3 \text { (month) }\end{array}$ \\
\hline Qiu $2006^{20}$ & $A C R$ & $30 / 30$ & $\begin{array}{l}\mathrm{T}: 5 / 25 \\
\mathrm{C}: 4 / 26\end{array}$ & $\begin{array}{l}\mathrm{T}: 56.07 \\
\mathrm{C}: 55.37\end{array}$ & $\begin{array}{l}\mathrm{T}: 8.95 \\
\mathrm{C}: 9.55\end{array}$ \\
\hline Gang2 $016^{21}$ & $\begin{array}{l}\text { Guidelines for the diagnosis and treatment of } \\
\text { osteoarthritis }\end{array}$ & $43 / 45$ & $\begin{array}{l}\mathrm{T}: 19 / 24 \\
\mathrm{C}: 22 / 23\end{array}$ & $\begin{array}{l}\mathrm{T}: 54 \pm 8 \\
\mathrm{C}: 54 \pm 8\end{array}$ & $\begin{array}{l}\mathrm{T}: I .1 \pm 0.6 \\
\mathrm{C}: 1.2 \pm 0.6\end{array}$ \\
\hline Gao $2011^{22}$ & $A C R$ & $34 / 35$ & $\begin{array}{l}T: 13 / 2 \mid \\
C: 15 / 20\end{array}$ & $\begin{array}{l}\mathrm{T}: 57.68 \pm 8.67 \\
\mathrm{C}: 58.57 \pm 8.89\end{array}$ & $\begin{array}{l}\mathrm{T}: 37.35 \pm 10.83 \text { (month) } \\
\mathrm{C}: 38.86 \pm 12.12 \text { (month) }\end{array}$ \\
\hline Wang $2017^{23}$ & Diagnostic criteria for blood stasis type of KOA & $48 / 47$ & $\begin{array}{l}\mathrm{T}: 25 / 23 \\
\mathrm{C}: 24 / 23\end{array}$ & $\begin{array}{l}T: 56.15 \pm 7.36 \\
C: 56.19 \pm 7.33\end{array}$ & - \\
\hline Yin $2017^{24}$ & $\begin{array}{l}\text { Guidelines for the diagnosis and treatment of } \\
\text { osteoarthritis }\end{array}$ & $60 / 60$ & - & - & - \\
\hline Ju $2017^{25}$ & $A C R$ & $30 / 30$ & $\begin{array}{l}\mathrm{T}: 6 / 24 \\
\mathrm{C}: 7 / 23\end{array}$ & $\begin{array}{l}\mathrm{T}: 60 \pm 10 \\
\mathrm{C}: 64 \pm 6\end{array}$ & $\begin{array}{l}\mathrm{T}: 29.89 \pm 29.74 \text { (month) } \\
\mathrm{C}: 32.74 \pm 31.43 \text { (month) }\end{array}$ \\
\hline Wu $2012^{26}$ & $A C R$ & $30 / 30$ & $\begin{array}{l}\mathrm{T}: 11 / 19 \\
\mathrm{C}: 13 / 17\end{array}$ & $\begin{array}{l}T: 60.63 \pm 6.44 \\
C: 59.87 \pm 6.77\end{array}$ & $\begin{array}{l}\mathrm{T}: 3.47 \pm 1.27 \\
\mathrm{C}: 3.23 \pm 1.48\end{array}$ \\
\hline Chen $2018^{27}$ & $\begin{array}{l}\text { Guidelines for the diagnosis and treatment of } \\
\text { osteoarthritis }\end{array}$ & $45 / 45$ & $\begin{array}{l}T: 31 / 14 \\
C: 28 / 17\end{array}$ & $\begin{array}{l}\mathrm{T}: 65.5 \mathrm{I} \pm 3.26 \\
\mathrm{C}: 66.36 \pm 3.08\end{array}$ & $\begin{array}{l}\mathrm{T}: 3.56 \pm 1.87 \\
\mathrm{C}: 3.49 \pm 1.76\end{array}$ \\
\hline Liu $2014^{28}$ & $A C R$ & $30 / 30$ & $\begin{array}{l}\mathrm{T}: 10 / 20 \\
\mathrm{C}: 12 / 18\end{array}$ & $\begin{array}{l}\mathrm{T}: 58.07 \pm 8.76 \\
\mathrm{C}: 59.47 \pm 7.92\end{array}$ & - \\
\hline $\operatorname{Tan} 2016^{29}$ & $\begin{array}{l}\text { Guidelines for the diagnosis and treatment of } \\
\text { osteoarthritis }\end{array}$ & $40 / 40$ & $\begin{array}{l}T: 18 / 22 \\
C: 17 / 23\end{array}$ & $\begin{array}{l}\mathrm{T}: 52.72 \pm 5.83 \\
\mathrm{C}: 51.93 \pm 6.18\end{array}$ & $\begin{array}{l}\mathrm{T}: 1-10 \\
\mathrm{C}: \mathrm{I}-12\end{array}$ \\
\hline
\end{tabular}


Table 2 (Continued).

\begin{tabular}{|c|c|c|c|c|c|}
\hline Included Studies & Diagnostic Criteria & $\begin{array}{l}\text { Sample } \\
\text { Size (T/ } \\
\text { C) }\end{array}$ & $\begin{array}{l}\text { Sex } \\
\text { (Men/ } \\
\text { Women) }\end{array}$ & Age (Year) & $\begin{array}{l}\text { Course of Disease } \\
\text { (Year) }\end{array}$ \\
\hline Ma $2015^{30}$ & $\begin{array}{l}\text { Guidelines for the diagnosis and treatment of } \\
\text { osteoarthritis }\end{array}$ & $54 / 54$ & $\begin{array}{l}\mathrm{T}: 22 / 32 \\
\mathrm{C}: 19 / 35\end{array}$ & $\begin{array}{l}\mathrm{T}: 53.2 \pm 7.5 \\
\mathrm{C}: 56.3 \pm 8.6\end{array}$ & $\begin{array}{l}\mathrm{T}: 3.2 \pm \mathrm{I} .4 \\
\mathrm{C}: 4.4 \pm \mathrm{I} .5\end{array}$ \\
\hline Chen $2012^{31}$ & $A C R$ & $30 / 30$ & $\begin{array}{l}\mathrm{T}: 8 / 22 \\
\mathrm{C}: 9 / 21\end{array}$ & $\begin{array}{l}T: 59 \pm 12 \\
C: 59 \pm 16\end{array}$ & $\begin{array}{l}\mathrm{T}: 50.23 \pm 47.50 \text { (month) } \\
\mathrm{C}: 62.20 \pm 45.67 \text { (month) }\end{array}$ \\
\hline Jiang $2013^{32}$ & $A C R$ & $42 / 44$ & $\begin{array}{l}\mathrm{T}: 18 / 24 \\
\mathrm{C}: 21 / 23\end{array}$ & $\begin{array}{l}\mathrm{T}: 65.7 \pm 3.9 \\
\mathrm{C}: 67.7 \pm 4.5\end{array}$ & $\begin{array}{l}\mathrm{T}: 3.7 \pm 0.9 \\
\mathrm{C}: 4.2 \pm 1.5\end{array}$ \\
\hline Ren $2018^{33}$ & $A C R$ & $54 / 54$ & $\begin{array}{l}\mathrm{T}: 23 / 31 \\
\mathrm{C}: 25 / 29\end{array}$ & $\begin{array}{l}\mathrm{T}: 67.1 \pm 4.6 \\
\mathrm{C}: 68.7 \pm 5.2\end{array}$ & $\begin{array}{l}\mathrm{T}: 4.9 \pm 1.5 \\
\mathrm{C}: 4.6 \pm 1.7\end{array}$ \\
\hline Zhang $2016^{34}$ & $\begin{array}{l}\text { Standard of diagnosis and curative effect of disease } \\
\text { and syndrome in TCM }\end{array}$ & $30 / 30$ & $\begin{array}{l}\mathrm{T}: 18 / 12 \\
\mathrm{C}: 19 / 11\end{array}$ & $\begin{array}{l}\mathrm{T}: 56.0 \pm 2.9 \\
\mathrm{C}: 55.9 \pm 2.8\end{array}$ & $\begin{array}{l}\mathrm{T}: 5.6 \pm 0.5 \\
\mathrm{C}: 5.7 \pm 0.5\end{array}$ \\
\hline $\operatorname{Pan} 2020^{35}$ & $A C R$ & $35 / 35$ & $\begin{array}{l}\mathrm{T}: 11 / 24 \\
\mathrm{C}: 9 / 26\end{array}$ & $\begin{array}{l}\mathrm{T}: 65.086 \\
\mathrm{C}: 64.2\end{array}$ & $\begin{array}{l}\mathrm{T}: 3.34 \pm 1.72 \\
\mathrm{C}: 3.03 \pm 1.33\end{array}$ \\
\hline Ji $2012^{36}$ & $\begin{array}{l}\text { Guiding principles of clinical research on new chinese } \\
\text { medicine }\end{array}$ & $30 / 30$ & $\begin{array}{l}\mathrm{T}: 12 / 18 \\
\mathrm{C}: 11 / 19\end{array}$ & $\begin{array}{l}\mathrm{T}: 56.73 \pm 10.08 \\
\mathrm{C}: 58.77 \pm 7.98\end{array}$ & $\begin{array}{l}\mathrm{T}: 38.17 \pm 23.99 \text { (month) } \\
\mathrm{C}: 39.23 \pm 25.54 \text { (month) }\end{array}$ \\
\hline Shen $2017^{37}$ & $\begin{array}{l}\text { Standard of diagnosis and curative effect of disease } \\
\text { and syndrome in TCM }\end{array}$ & $100 / 100$ & $\begin{array}{l}\mathrm{T}: 54 / 46 \\
\mathrm{C}: 5 \mathrm{I} / 49\end{array}$ & $\begin{array}{l}\mathrm{T}: 66.12 \pm 2.09 \\
\mathrm{C}: 66.5 \mathrm{I} \pm 2.12\end{array}$ & $\begin{array}{l}\mathrm{T}: 3.31 \pm 0.23 \\
\mathrm{C}: 3.35 \pm 0.12\end{array}$ \\
\hline Miao $2014^{38}$ & $\begin{array}{l}\text { Guidelines for the diagnosis and treatment of } \\
\text { osteoarthritis }\end{array}$ & $35 / 35 / 35$ & $\begin{array}{l}\mathrm{T}: 5 / 30 \\
\mathrm{Cl}: 9 / 26 \\
\mathrm{C} 2: 7 / 28\end{array}$ & $\begin{array}{l}\mathrm{T}: 57.5 \pm 9.7 \\
\mathrm{C} 1: 56.3 \pm 8.9 \\
\mathrm{C} 2: 60.4 \pm 10.5\end{array}$ & $\begin{array}{l}T: 5.6 \pm 3.8 \\
C 1: 6.4 \pm 4.2 \\
C 2: 6.8 \pm 3.3\end{array}$ \\
\hline Zhang $2016^{39}$ & $\begin{array}{l}\text { Guidelines for the diagnosis and treatment of } \\
\text { arthritis }\end{array}$ & $28 / 28$ & $\begin{array}{l}T: 11 / 17 \\
C: 12 / 16\end{array}$ & $\begin{array}{l}\mathrm{T}: 62.3 \pm 5.1 \\
\mathrm{C}: 61.8 \pm 4.7\end{array}$ & $\begin{array}{l}\mathrm{T}: 8.7 \pm 3.6 \\
\mathrm{C}: 8.4 \pm 3.4\end{array}$ \\
\hline $\operatorname{Lin} 2018^{40}$ & $\begin{array}{l}\text { The National Institute for Health and Clinical } \\
\text { Excellence Guidelines } 2014 \text { Edition criteria }\end{array}$ & $21 / 21$ & $\begin{array}{l}\mathrm{T}: 4 / 17 \\
\mathrm{C}: 1 / 20\end{array}$ & $\begin{array}{l}\mathrm{T}: 59.5 \pm 7.5 \\
\mathrm{C}: 60.0 \pm 7.3\end{array}$ & $\begin{array}{l}\mathrm{T}: 60.0 \pm 45.9 \text { (month) } \\
\mathrm{C}: 63.1 \pm 45.4 \text { (month) }\end{array}$ \\
\hline Zhao $2014^{41}$ & $A C R$ & $55 / 55$ & $\begin{array}{l}T: 16 / 39 \\
\mathrm{C}: 21 / 34\end{array}$ & $\begin{array}{l}\mathrm{T}: 65.80 \pm 7.45 \\
\mathrm{C}: 64.55 \pm 8.38\end{array}$ & - \\
\hline Zhang $2013^{42}$ & $A C R$ & $33 / 34$ & $\begin{array}{l}\mathrm{T}: 13 / 20 \\
\mathrm{C}: 14 / 20\end{array}$ & $\begin{array}{l}\mathrm{T}: 57 \pm 8 \\
\mathrm{C}: 58 \pm 9\end{array}$ & $\begin{array}{l}\mathrm{T}: 38 \pm 10 \text { (month) } \\
\mathrm{C}: 38 \pm 1 \mathrm{I} \text { (month) }\end{array}$ \\
\hline Zheng $2016^{43}$ & $A C R$ & $35 / 35$ & $\begin{array}{l}T: 16 / 19 \\
C: 15 / 20\end{array}$ & $\begin{array}{l}\mathrm{T}: 62.39 \pm 8.004 \\
\mathrm{C}: 6 \mathrm{I} .4 \mathrm{I} \pm 8.203\end{array}$ & $\begin{array}{l}\mathrm{T}: 135.97 \pm 74.068 \text { (month) } \\
\mathrm{C}: 128.03 \pm 70.194 \text { (month) }\end{array}$ \\
\hline Lu $201 I^{44}$ & $A C R$ & $27 / 27$ & $\begin{array}{l}\mathrm{T}: 6 / 21 \\
\mathrm{C}: 8 / 19\end{array}$ & $\begin{array}{l}T: 54.11 \pm 9.46 \\
C: 60.8 I \pm 10.09\end{array}$ & $\begin{array}{l}\mathrm{T}: 3.83 \pm 5.78 \\
\mathrm{C}: 3.69 \pm 2.98\end{array}$ \\
\hline Li $2020^{45}$ & $\begin{array}{l}\text { Guidelines for the diagnosis and treatment of } \\
\text { osteoarthritis }\end{array}$ & $36 / 36$ & $\begin{array}{l}\mathrm{T}: 12 / 24 \\
\mathrm{C}: 14 / 22\end{array}$ & $\begin{array}{l}\mathrm{T}: 58.54 \pm 8.4 \mathrm{I} \\
\mathrm{C}: 56.52 \pm 7.9 \mathrm{I}\end{array}$ & - \\
\hline Tang $2017^{46}$ & $\begin{array}{l}\text { Guidelines for the diagnosis and treatment of } \\
\text { osteoarthritis }\end{array}$ & $30 / 30$ & $\begin{array}{l}\mathrm{T}: 8 / 22 \\
\mathrm{C}: 9 / 21\end{array}$ & $\begin{array}{l}\mathrm{T}: 59.64 \\
\mathrm{C}: 60.40\end{array}$ & $\begin{array}{l}\mathrm{T}: 2.62 \\
\mathrm{C}: 2.73\end{array}$ \\
\hline Song $2020^{47}$ & $\begin{array}{l}\text { Guidelines for the diagnosis and treatment of } \\
\text { osteoarthritis }\end{array}$ & $30 / 30$ & $\begin{array}{l}\mathrm{T}: 12 / 18 \\
\mathrm{C}: 14 / 16\end{array}$ & $\begin{array}{l}\mathrm{T}: 53.83 \pm 5.37 \\
\mathrm{C}: 53.47 \pm 7.34\end{array}$ & $\begin{array}{l}\mathrm{T}: 37.90 \pm 16.01 \text { (month) } \\
\mathrm{C}: 34.67 \pm 19.78 \text { (month) }\end{array}$ \\
\hline $\operatorname{Lin} 2012^{48}$ & $\begin{array}{l}\text { Guidelines for the diagnosis and treatment of } \\
\text { osteoarthritis }\end{array}$ & $30 / 30$ & $\begin{array}{l}T: 14 / 16 \\
C: 16 / 14\end{array}$ & $\begin{array}{l}\mathrm{T}: 48.47 \pm \mathrm{I} 1.95 \\
\mathrm{C}: 50.07 \pm 9.7\end{array}$ & $\begin{array}{l}\mathrm{T}: 4.97 \pm 7.1 \text { (month) } \\
\mathrm{C}: 9.83 \pm 17.74 \text { (month) }\end{array}$ \\
\hline
\end{tabular}

(Continued) 
Table 2 (Continued).

\begin{tabular}{|c|c|c|c|c|c|}
\hline Included Studies & Diagnostic Criteria & $\begin{array}{l}\text { Sample } \\
\text { Size (T/ } \\
\text { C) }\end{array}$ & $\begin{array}{l}\text { Sex } \\
\text { (Men/ } \\
\text { Women) }\end{array}$ & Age (Year) & $\begin{array}{l}\text { Course of Disease } \\
\text { (Year) }\end{array}$ \\
\hline $\mathrm{He} 2018^{49}$ & $A C R$ & $57 / 55$ & $\begin{array}{l}\mathrm{T}: 26 / 31 \\
\mathrm{C}: 22 / 33\end{array}$ & $\begin{array}{l}T: 56 \pm 5 \\
C: 58 \pm 5\end{array}$ & $\begin{array}{l}\mathrm{T}: 73.28 \pm 29.24 \text { (month) } \\
\mathrm{C}: 71.09 \pm 27.96 \text { (month) }\end{array}$ \\
\hline Fan $2016^{50}$ & $\begin{array}{l}\text { Guidelines for the diagnosis and treatment of } \\
\text { osteoarthritis }\end{array}$ & $54 / 54$ & $\begin{array}{l}\mathrm{T}: 33 / 21 \\
\mathrm{C}: 30 / 24\end{array}$ & $\begin{array}{l}\mathrm{T}: 58 \pm 6.2 \\
\mathrm{C}: 56 \pm 8.4\end{array}$ & $\begin{array}{l}\mathrm{T}: 14.8 \pm 8.8 \text { (month) } \\
\mathrm{C}: 12.7 \pm \\
7.3 \text { (month) }\end{array}$ \\
\hline
\end{tabular}

Abbreviations: ACR, American College of Rheumatology; T, treatment group; C, control group; -, not mentioned.

sealed opaque envelopes, one study ${ }^{40}$ used identical and ordered drug containers, one study ${ }^{41}$ used alphabetic codes, while the remaining thirty-five studies did not mention allocation concealment; iii. Blinding of patients and experimentalists: Four studies ${ }^{13,14,40,41}$ blinded patients and experimentalists; iv. Blinding of outcome evaluators: Five studies $^{13,14,36,40,41}$ blinded outcome evaluators; v. Incomplete result data, selective reporting, other bias: The results of 40 studies ${ }^{11-50}$ were all complete, without selective reporting and other bias. Results of bias risk assessment are shown in Figure 2.

\section{Directly Compared Meta-Analysis Results Visual Analogue Scale}

Results of the meta-analysis showed that the VAS score of the moxibustion group was significantly better than that of the western medicine group (4 RCTs; SMD 0.624, 95\% CI 1.239 to $0.009 ; I^{2}=82.10 \%, P=0.001$ ), whereas the VAS score of the western medicine group was significantly better than that of the electro-acupuncture group (6 RCTs; SMD $1.201,95 \%$ CI 0.169 to $2.223 ; I^{2}=95.80 \%$, $P<0.00001)$. The VAS score of conventional acupuncture group was significantly better than that of warm needle group (3 RCTs; SMD 2.974, 95\% CI 0.798 to $5.150 ; I^{2}$ $=97.60 \%, P<0.00001)$ and fire needle group ( 3 RCTs; SMD $1.22,95 \%$ CI 0.681 to $1.760 ; I^{2}=67.5 \%, P=0.046$ ), and the VAS score of warm needle group was substantially better than that of fire needle group (3 RCTs; SMD 1.432, 95\% CI 0.669 to $\left.2.194 ; I^{2}=80.90 \%, P=0.005\right)(P<0.05)$. Descriptive analysis results showed that VAS score of electro-acupuncture group was significantly better than that of conventional acupuncture group, and VAS score of conventional acupuncture group was significantly better than that of sham acupuncture group. The VAS score of western medicine group was significantly better than that of fire needle group $(P<0.05)$. There was no significant difference among other interventions in the aforementioned scores $(P>0.05)$. See supplementary materials (Table S2).

\section{WOMAC Pain Score}

Meta-analysis results showed that the WOMAC pain score of the western medicine group was significantly better than that of the warm needle group (4 RCTs; SMD 0.664, 95\% CI 0.306 to $1.022 ; I^{2}=62.10 \%, P=0.048$ ), while the WOMAC pain score of the warm needle group was significantly better than that of the fire needle group (3 RCTs; SMD 0.956 , 95\% CI 0.139 to $1.774 ; I^{2}=88.10 \%$, $P<0.00001)(P<0.05)$. Descriptive analysis results showed that WOMAC pain score of electro-acupuncture group was significantly better than that of conventional acupuncture group, and WOMAC pain score of conventional acupuncture group was significantly better than that of warm needle group and that of the fire needle group $(P<0.05)$. There was no differences among other interventions in the aforementioned scores, $(P>0.05)$. See supplementary materials (Table S1).

\section{WOMAC Joint Function Score}

Meta-analysis results showed that the WOMAC joint function score of the western medicine group was significantly better than that of the electro-acupuncture group (4 RCTs; SMD $0.419,95 \%$ CI 0.209 to $0.629 ; I^{2}=20.90 \%, P=0.285$ ) and that of the warm needle group (4 RCTs; SMD 0.646, 95\% CI 0.201 to $\left.1.091 ; I^{2}=75.40 \%, P=0.007\right)(P<0.05)$. Descriptive analysis results showed that WOMAC joint function score of moxibustion group was significantly better than that of western medicine group, and WOMAC joint function score of western medicine group was significantly better than that of conventional acupuncture group, and WOMAC joint function score of electro-acupuncture group 
Table 3 Characteristics of Interventions

\begin{tabular}{|c|c|c|c|c|c|}
\hline \multirow{2}{*}{$\begin{array}{l}\text { Included } \\
\text { Studies }\end{array}$} & \multirow[t]{2}{*}{ Study Type } & \multicolumn{2}{|l|}{ Interventions } & \multirow{2}{*}{$\begin{array}{l}\text { Course of } \\
\text { Treatment } \\
\text { (Week) }\end{array}$} & \multirow{2}{*}{$\begin{array}{l}\text { Outcome } \\
\text { Indicators }\end{array}$} \\
\hline & & Treatment Group & Control Group & & \\
\hline Zhang 2011"1 & Double-arm & Moxibustion & celecoxib $200 \mathrm{mg}, \mathrm{I} / \mathrm{d}$ & 6 & $\mathrm{~d}, \mathrm{e}$ \\
\hline Zhou $2014^{12}$ & Three-arm & Moxibustion & $\begin{array}{l}\mathrm{Cl} \text { :electro-acupuncture } \\
\mathrm{C} 2 \text { :celecoxib } 200 \mathrm{mg}, \mathrm{I} / \mathrm{d}\end{array}$ & 4 & $d$ \\
\hline Zhou $2017^{13}$ & Double-arm & Moxibustion & Diclofenac sodium gel & 4 & $a, b, c, d$ \\
\hline $\operatorname{Ren} 2011^{14}$ & Double-arm & Moxibustion & Sham moxibustion & 6 & $a, b, c$ \\
\hline Zhou $2015^{15}$ & Double-arm & Electro-acupuncture & $\begin{array}{l}\text { Diclofenac sodium sustained release capsules } 50 \mathrm{mg} \text {, } \\
\mathrm{I} / \mathrm{d}\end{array}$ & 4 & $d$ \\
\hline Zhang $2018^{16}$ & Double-arm & Electro-acupuncture & Meloxicam dispersible tablets $7.5 \mathrm{mg}, \mathrm{I} / \mathrm{d}$ & 2 & $d$ \\
\hline Liu $2020^{17}$ & Double-arm & Electro-acupuncture & Conventional acupuncture & 4 & d \\
\hline Guo $2016^{18}$ & Double-arm & Electro-acupuncture & $\begin{array}{l}\text { Diclofenac sodium double release enteric capsules } 75 \\
\mathrm{mg}, \mathrm{I} / \mathrm{d}\end{array}$ & 3 & $a, b, c$ \\
\hline Wu $2015^{19}$ & Double-arm & Electro-acupuncture & Votalin emulsion & 4 & $d$ \\
\hline Qiu $2006^{20}$ & Double-arm & Electro-acupuncture & Futarin sustained-release tablets $75 \mathrm{mg}, \mathrm{I} / \mathrm{d}$ & 4 & $a, b, c, e$ \\
\hline Gang $2016^{21}$ & Double-arm & Electro-acupuncture & Meloxicam tablets $7.5 \mathrm{mg}, \mathrm{I} / \mathrm{d}$ & 6 & $a, b, c$ \\
\hline Gao $201 I^{22}$ & Double-arm & Electro-acupuncture & Warm needle & 8 & $a, b, c, d$ \\
\hline Wang $2017^{23}$ & Double-arm & Electro-acupuncture & Warm needle & 3 & $d$ \\
\hline Yin $2017^{24}$ & Double-arm & Electro-acupuncture & Glucosamine $240 \mathrm{mg}, \mathrm{I} / \mathrm{d}$ & 8 & $a, b, e$ \\
\hline Ju $2017^{25}$ & Double-arm & Electro-acupuncture & Celecoxib capsules $200 \mathrm{mg}, \mathrm{I} / \mathrm{d}$ & 2 & $a, b, c, d$ \\
\hline Wu $2012^{26}$ & Double-arm & Electro-acupuncture & Conventional acupuncture & 4 & $a, b, c$ \\
\hline Chen $2018^{27}$ & Double-arm & Warm needle & $\begin{array}{l}\text { Glucosamine sulfate capsules } 314 \mathrm{mg}, 2 \text { capsules/ } \\
\text { times, } 3 \text { times/ d }\end{array}$ & 4 & $a, b, c$ \\
\hline Liu $2014^{28}$ & Double-arm & Warm needle & Electro-acupuncture & 4 & $a, b, c$ \\
\hline $\operatorname{Tan} 2016^{29}$ & Double-arm & Warm needle & Conventional acupuncture & 3 & $d$ \\
\hline Ma $2015^{30}$ & Double-arm & Warm needle & Conventional acupuncture & 4 & $d$ \\
\hline Chen $2012^{31}$ & Double-arm & Warm needle & $\begin{array}{l}\text { Ibuprofen sustained release capsules } 300 \mathrm{mg}, 2 \text { times/ } \\
\text { d }\end{array}$ & 3 & $\mathrm{a}, \mathrm{b}, \mathrm{c}, \mathrm{e}$ \\
\hline Jiang $2013^{32}$ & Double-arm & Warm needle & $\begin{array}{l}\text { Glucosamine sulfate tablets } 314 \mathrm{mg}, 2 \text { tablets/times, } 3 \\
\text { times/d }\end{array}$ & 8 & a,b.c.e \\
\hline Ren $2018^{33}$ & Double-arm & Warm needle & $\begin{array}{l}\text { Glucosamine hydrochloride tablets } 240 \mathrm{mg}, 2 \text { tablets/ } \\
\text { times, } 3 \text { times } / \mathrm{d}\end{array}$ & 20 & $a, b, c$ \\
\hline Zhang $2016^{34}$ & Double-arm & Warm needle & Conventional acupuncture & 4 & $a, b, c, d$ \\
\hline Pan $2020^{35}$ & Double-arm & $\begin{array}{l}\text { Acupoint catgut } \\
\text { embedding }\end{array}$ & Conventional acupuncture & 3 & $d$ \\
\hline Ji $2012^{36}$ & Double-arm & Electro-acupuncture & Warm needle & 8 & $a, b, c, d$ \\
\hline
\end{tabular}

(Continued) 
Table 3 (Continued).

\begin{tabular}{|c|c|c|c|c|c|}
\hline \multirow{2}{*}{$\begin{array}{l}\text { Included } \\
\text { Studies }\end{array}$} & \multirow[t]{2}{*}{ Study Type } & \multicolumn{2}{|l|}{ Interventions } & \multirow{2}{*}{$\begin{array}{l}\text { Course of } \\
\text { Treatment } \\
\text { (Week) }\end{array}$} & \multirow{2}{*}{$\begin{array}{l}\text { Outcome } \\
\text { Indicators }\end{array}$} \\
\hline & & Treatment Group & Control Group & & \\
\hline Shen $2017^{37}$ & Double-arm & $\begin{array}{l}\text { Conventional } \\
\text { acupuncture }\end{array}$ & Diclofenac sodium emulsion & 4 & $b, c, e$ \\
\hline Miao $2014^{38}$ & Three-arm & Moxibustion & $\begin{array}{l}\mathrm{Cl} \text { : electro-acupuncture } \\
\mathrm{C} 2 \text { : celecoxib capsules } 200 \mathrm{mg}, \mathrm{I} / \mathrm{d}\end{array}$ & 4 & $d$ \\
\hline Zhang $2016^{39}$ & Double-arm & $\begin{array}{l}\text { Conventional } \\
\text { acupuncture }\end{array}$ & Warm needle & 4 & e \\
\hline $\operatorname{Lin} 2018^{40}$ & Double-arm & $\begin{array}{l}\text { Conventional } \\
\text { acupuncture }\end{array}$ & Sham acupuncture & 26 & $a, b, d, e$ \\
\hline Zhao $2014^{41}$ & Double-arm & Moxibustion & Sham moxibustion & 6 & $a, c, e$ \\
\hline Zhang $2013^{42}$ & Double-arm & Fire needle & Warm needle & 4 & $a, b, c, d$ \\
\hline Guo $2016^{43}$ & Double-arm & Fire needle & Conventional acupuncture & 3 & $a, b, d$ \\
\hline Lu $201 I^{44}$ & Double-arm & Fire needle & Warm needle & 4 & d,e \\
\hline Li $2020^{45}$ & Double-arm & Fire needle & Conventional acupuncture & 24 & $d$ \\
\hline Tang $2017^{46}$ & Double-arm & Fire needle & Warm needle & 4 & $a, b, c, d$ \\
\hline Song $2020^{47}$ & Double-arm & Fire needle & $\begin{array}{l}\text { Celecoxib capsules } 200 \mathrm{mg}, \mathrm{I} / \mathrm{d} \text {; diclofenac sodium } \\
\text { diethylamine emulsion agent } 200 \mathrm{mg}, 3 \text { times } / \mathrm{d}\end{array}$ & 3 & $d$ \\
\hline $\operatorname{Lin} 2012^{48}$ & Double-arm & Fire needle & Conventional acupuncture & 4 & d,e \\
\hline $\mathrm{He} 2018^{49}$ & Double-arm & Electro-acupuncture & Fire needle & 4 & a,b,c,d,e \\
\hline Fan $2016^{50}$ & Double-arm & Fire needle & Warm needle & 4 & $\mathrm{a}$ \\
\hline
\end{tabular}

Notes: a, WOMAC pain scores; b, WOMAC stiffness scores; c, WOMAC joint function scores; d, VAS scores; e. Adverse events.

was significantly better than that of conventional acupuncture group, and WOMAC joint function score of conventional acupuncture group was significantly better than that of warm needle group, and the differences were statistically significant $(P<0.05)$. There was no statistical significance in the comparison of other interventions $(P>0.05)$. See supplementary materials (Table S1).

\section{WOMAC Stiffness Score}

Meta-analysis results showed that the WOMAC stiffness score of the western medicine group was significantly better than that of the electro-acupuncture group (6 RCTs; SMD $1.201,95 \%$ CI 0.169 to $2.223 ; I^{2}=95.80 \%, P<0.00001$ ), while the WOMAC stiffness score of the electro-acupuncture group was significantly better than that of the warm needle (3 RCTs; SMD $0.671,95 \%$ CI 0.377 to $0.965 ; I^{2}=1.30 \%$, $P=0.363) \quad(P<0.05)$. Descriptive analysis results showed that WOMAC stiffness score of moxibustion group was significantly better than that of sham moxibustion group, and WOMAC stiffness score of western medicine group and electro-acupuncture group were significantly better than that of conventional acupuncture group, respectively, and WOMAC stiffness score of conventional acupuncture group was significantly better than that of warm needle group and that of fire needle group, respectively $(P<0.05)$. There was no significant difference in the aforementioned scores among other interventions $(P>0.05)$. See supplementary materials (Table S1).

\section{Heterogeneity Analysis}

In the direct comparison meta-analysis, most results were heterogeneous. Through the analysis of original data, we found that the lack of description of blind methods and allocation concealment in included studies may lead to methodological heterogeneity, at the same time, the inclusion of population, KOA stage and other factors may cause clinical 
heterogeneity, but since the original study did not specify these details and the number of included studies was small, further subgroup analysis could not be performed to explore the source of heterogeneity. However, the sensitivity analysis of the study results by a one-by-one exclusion method showed that the results were stable after the exclusion of any study. Therefore, we can ignore this heterogeneity and use a random effects model to analyze the results.

\section{Comparison Results of Network Meta- Analysis Evidence Network Diagram}

Twenty-three studies $^{13,14,18,20-22,24-28,31-34,36,40-43,46,49,50}$ reported WOMAC pain scores, forming five closed loops, that is electro-acupuncture-conventional acupuncture-warm needle, western medicine-electro-acupuncture-warm needle, electro-acupuncture-conventional acupuncture-fire needle, fire needle-conventional acupuncture-warm needle and electro-acupuncture-warm needle-fire needle; Twenty-one studies $^{13,14,18,20-22,24-28,31-34,36,37,42,43,46,49}$ reported WOMAC stiffness scores, forming seven closed loops, that is western medicine-electro-acupuncture-conventional acupuncture, electro-acupuncture-conventional acupuncture-fire needle, electro-acupuncture-conventional acupuncture-warm needle, warm needle-fire needle-conventional acupuncture, western medicine-warm needle-conventional acupuncture, western medicine-electro-acupuncture-warm needle, electro-acupuncture-warm needle-fire needle; Twenty-one $\quad$ studies $^{13,14,18,20-22,25-28,31-34,36,37,40-42,46,49}$ reported WOMAC joint function scores, forming five closed loops, that is electro-acupuncture-conventional acupuncture-warm needle, western medicine-electro-acupuncture-conventional acupuncture, western medicineconventional acupuncture-warm needle, western medicineelectro-acupuncture-warm needle, electro-acupuncturewarm needle-fire needle; Twenty-five studies $^{11-13,15-}$ 17,19,22,23,25,29,30,34-36,38,40,42-49 reported VAS scores, forming six closed loops, that is moxibustion-western medicineelectro-acupuncture, electro-acupuncture-conventional acupuncture-warm needle, electro-acupuncture-conventional acupuncture-fire needle, fire needle-conventional acupuncture-warm needle, western medicine -electro-acupuncturefire needle, electro-acupuncture-warm needle-fire needle. The thicker the line between the two measures, the larger the number of pairable studies between the two measures, the larger the node, and the larger the study sample size of the

intervention

involved

(Figure 3Figure 4Figure 5Figure -6 ).

\section{Network Meta-Analysis of WOMAC Pain Scores}

WOMAC pain scores were reported in 23 studies.13,14,18,20-22,24-28,31-34,36,40-43,46,49,50 Convergence assessment revealed that PSRF values tended to 1 and results of the incongruity model were similar to those of the congruity model, indicating that the stability and consistency of the indicators were good, therefore, the MCMC congruity model was used for network meta-analysis of WOMAC pain scores. It was found that the warm needle was superior to conventional acupuncture and western medicine; fire needle was superior to western medicine, electro-acupuncture, conventional acupuncture, warm needle and sham acupuncture while electro-acupuncture was superior to conventional acupuncture. WOMAC pain scores among other different acupuncture treatments showed no significant statistical difference (Table 4). Treatment ranking of WOMAC pain scores were: fire needle $>$ warm needle $>$ electro-acupuncture $>$ western medicine $>$ moxibustion $>$ conventional acupuncture $>$ sham acupuncture $>$ sham moxibustion (Table 5).

\section{Network Meta-Analysis of WOMAC Stiffness Scores} WOMAC stiffness scores were reported in 21 studies.13,14,18,20-22,24-28,31-34,36,37,42,43,46,49 Convergence assessment showed that PSRF values tended to 1 , and the convergence effect was satisfactory, the results of the incongruity model were similar to those of the congruity model, without significant changes, indicating that the stability and consistency of the indicators were good, therefore, the MCMC congruity model was used for network meta-analysis of WOMAC stiffness scores. It was found that electro-acupuncture was superior to western medicine and sham moxibustion, fire needle and warm needle were superior to western medicine and sham moxibustion, the difference was statistically significant. There was no statistically significant difference in improving WOMAC stiffness scores between other acupuncture treatments (Table 6). In the ranking of conformance model analysis, fire needle $>$ warm needle $>$ electro-acupuncture $>$ conventional acupuncture $>$ western medicine $>$ moxibustion $>$ sham moxibustion (Table 7).

\section{Network Meta-Analysis of WOMAC Joint Function Scores}

WOMAC joint function scores were reported in 21 studies. ${ }^{13,14,18,20-22,25-28,31-34,36,37,40-42,46,49}$ According to 


$$
\frac{\mathbb{Z}}{\mathbb{Z}}
$$




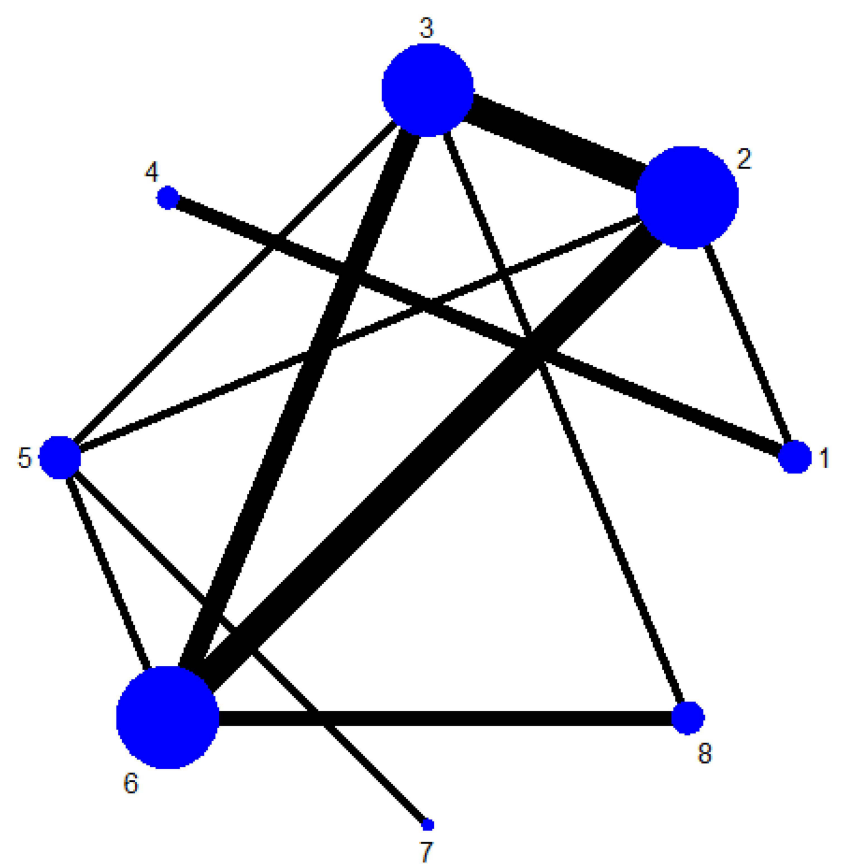

Figure 5 Evidence network diagram showing WOMAC joint function score for different acupuncture treatments for KOA.

Notes: I-moxibustion; 2-western medicine; 3-electro-acupuncture; 4-sham moxibustion; 5-conventional acupuncture; 6-warm needle; 7-sham acupuncture; 8-fire needle

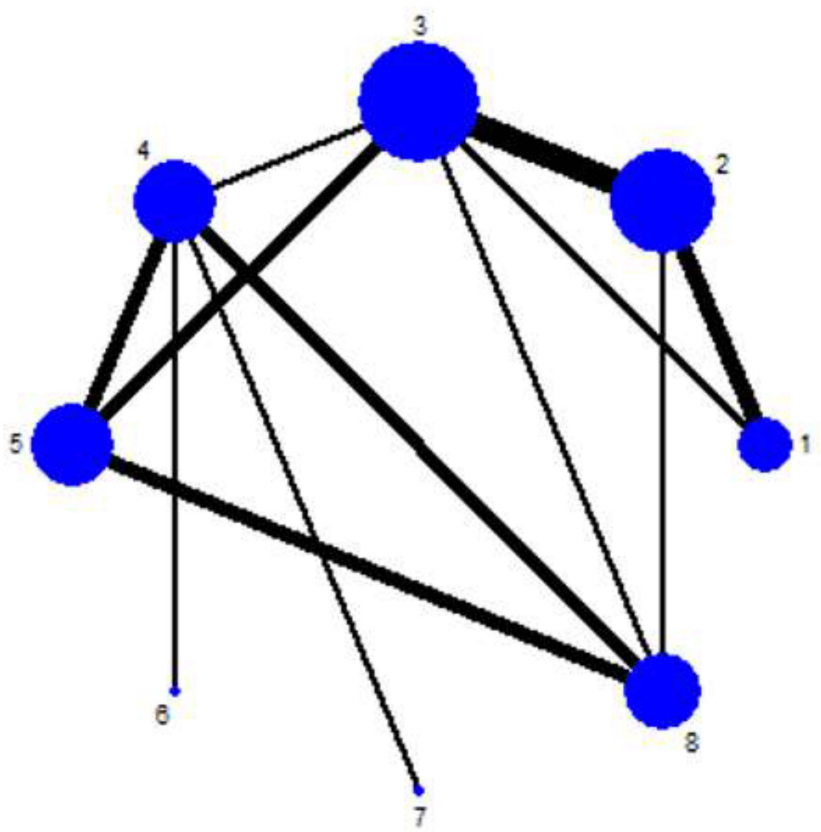

Figure 6 Evidence network diagram of VAS score for different acupuncture treatments for KOA.

Notes: I-moxibustion; 2-western medicine; 3-electro-acupuncture; 4-conventional acupuncture; 5-warm needle; 6-acupoint catgut embedding; 7-sham acupuncture; 8-fire needle

the congruity model, without significant changes, indicating that stability and consistency of the indicators were good, therefore, the MCMC congruity model was used for

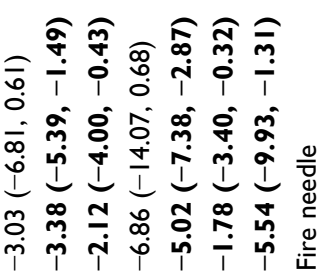

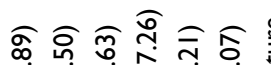

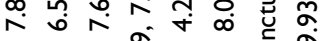

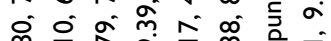
i i o I mo III工II $=$

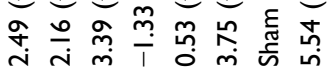

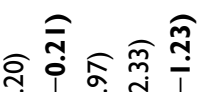

तิ

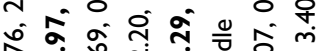
+ 1 T

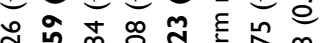
i i i

\begin{tabular}{|c|}
\hline 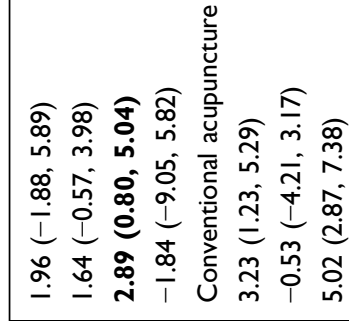 \\
\hline 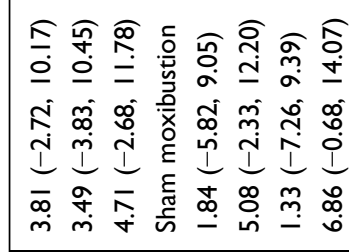 \\
\hline 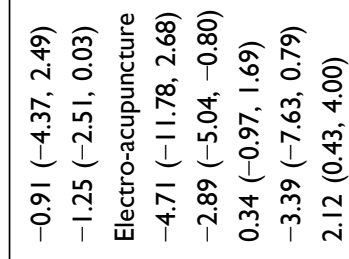 \\
\hline 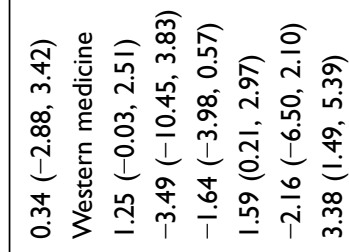 \\
\hline 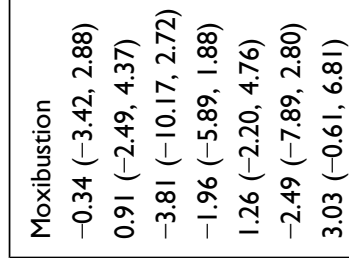 \\
\hline
\end{tabular}


Table 5 Ranking Probability Table of WOMAC Pain Scores

\begin{tabular}{|l|l|l|l|l|l|l|l|l|}
\hline Intervention & Rank I & Rank 2 & Rank 3 & Rank 4 & Rank 5 & Rank 6 & Rank 7 & Rank 8 \\
\hline Moxibustion & 0.01 & 0.09 & 0.13 & $\mathbf{0 . 2 5}$ & 0.24 & 0.11 & 0.13 & 0.04 \\
Western medicine & 0.01 & 0.04 & 0.19 & 0.45 & $\mathbf{0 . 3 1}$ & 0.01 & 0 & 0 \\
Electro-acupuncture & 0 & 0 & 0.01 & 0.06 & 0.22 & $\mathbf{0 . 5}$ & 0.21 & 0.01 \\
Sham moxibustion & $\mathbf{0 . 5 8}$ & 0.12 & 0.11 & 0.05 & 0.03 & 0.03 & 0.04 & 0.03 \\
Conventional acupuncture & 0.11 & 0.39 & $\mathbf{0 . 3 8}$ & 0.09 & 0.02 & 0 & 0 & 0 \\
Warm needle & 0 & 0 & 0 & 0.03 & 0.11 & 0.31 & $\mathbf{0 . 5 3}$ & 0.01 \\
Sham acupuncture & 0.29 & $\mathbf{0 . 3 6}$ & 0.18 & 0.08 & 0.06 & 0.02 & 0.02 & 0.01 \\
Fire needle & 0 & 0 & 0 & 0 & 0 & 0.01 & 0.07 & $\mathbf{0 . 9 1}$ \\
\hline
\end{tabular}

Notes: The bold font represents the probability of ordering the therapy.

network meta-analysis of WOMAC joint function scores. It was found that fire needle, warm needle and electroacupuncture yielded significantly better results in improving WOMAC joint function scores compared to conventional acupuncture and western medicine. There were no significant difference in the WOMAC joint function scores among the other acupuncture treatments (Table 8). In the ranking of conformance model analysis, fire needle $>$ electro-acupuncture $>$ warm needle $>$ moxibustion $>$ western medicine $>$ conventional acupuncture $>$ sham acupuncture $>$ sham moxibustion (Table 9).

\section{Network Meta-Analysis of VAS Scores}

VAS scores were reported in 25 studies. $^{11-13,15-}$ 17,19,22,23,25,29,30,34-36,38,40,42-49 Convergence assessment showed that PSRF values tended to 1 , and the convergence effect was satisfactory. Results of the incongruity model were similar to those of the congruity model, indicating that the stability and consistency of the indicators were good, therefore, the MCMC congruity model was used for network meta-analysis of VAS scores. The results showed that electro-acupuncture was superior to western medicine, conventional acupuncture and sham acupuncture while fire needle was superior to conventional acupuncture and sham acupuncture (Table 10). The probability ranking conducted with the MCMC method revealed that fire needle $>$ electro-acupuncture $>$ moxibustion $>$ warm needle $>$ western medicine $>$ conventional acupuncture $>$ acupoint catgut embedding $>$ sham acupuncture in improving VAS scores (Table 11).

\section{Small Sample Effect Estimation}

A comparative correction funnel plot was used to evaluate the outcome of the WOMAC pain scores (Figure 7). The funnel plot was not completely symmetric, suggesting that there may be some publication bias or small sample effect in the research network.

\section{Adverse Events}

Twelve studies ${ }^{11,20,24,31,32,37,39-41,44,48,49}$ reported the occurrence of adverse events (Table 12). Overall, there were only mild adverse reactions but no serious adverse reactions in the treatment of KOA by different acupuncture and moxibustion treatments.

\section{Discussion}

According to the first-line management protocol recommended by Osteoarthritis Research Society International (OARSI), conservative treatment (drug therapy and non-drug therapy) plays an important role in the management of osteoarthritis. ${ }^{51}$ However, due to adverse reactions which decrease patient compliance, the benefit-risk ratios of such interventions in KOA patients require urgent investigation. ${ }^{52,53}$ Traditional Chinese medicine classifies KOA as "bi zheng" (arthralgia syndrome). In China, acupuncture and moxibustion therapies have been used in the treatment of arthralgia syndrome for thousands of years, and this in ancient period in the Chinese book Lingshu. Acupuncture and moxibustion therapies are widely used in clinical practice and have high efficacy and strong safety. ${ }^{54,55}$ Studies have shown that these therapies can improve the pain threshold by promoting the release of analgesic substances in KOA patients. ${ }^{56}$

Herein, the effects of acupuncture and moxibustion therapy on WOMAC pain, stiffness, joint function scores and VAS scores in KOA patients were investigated. Results showed that warm needle was superior to conventional acupuncture and western medicine, fire needle was superior to western medicine, electro-acupuncture, conventional acupuncture, warm needle and sham acupuncture, while electro-acupuncture was superior to conventional acupuncture in 

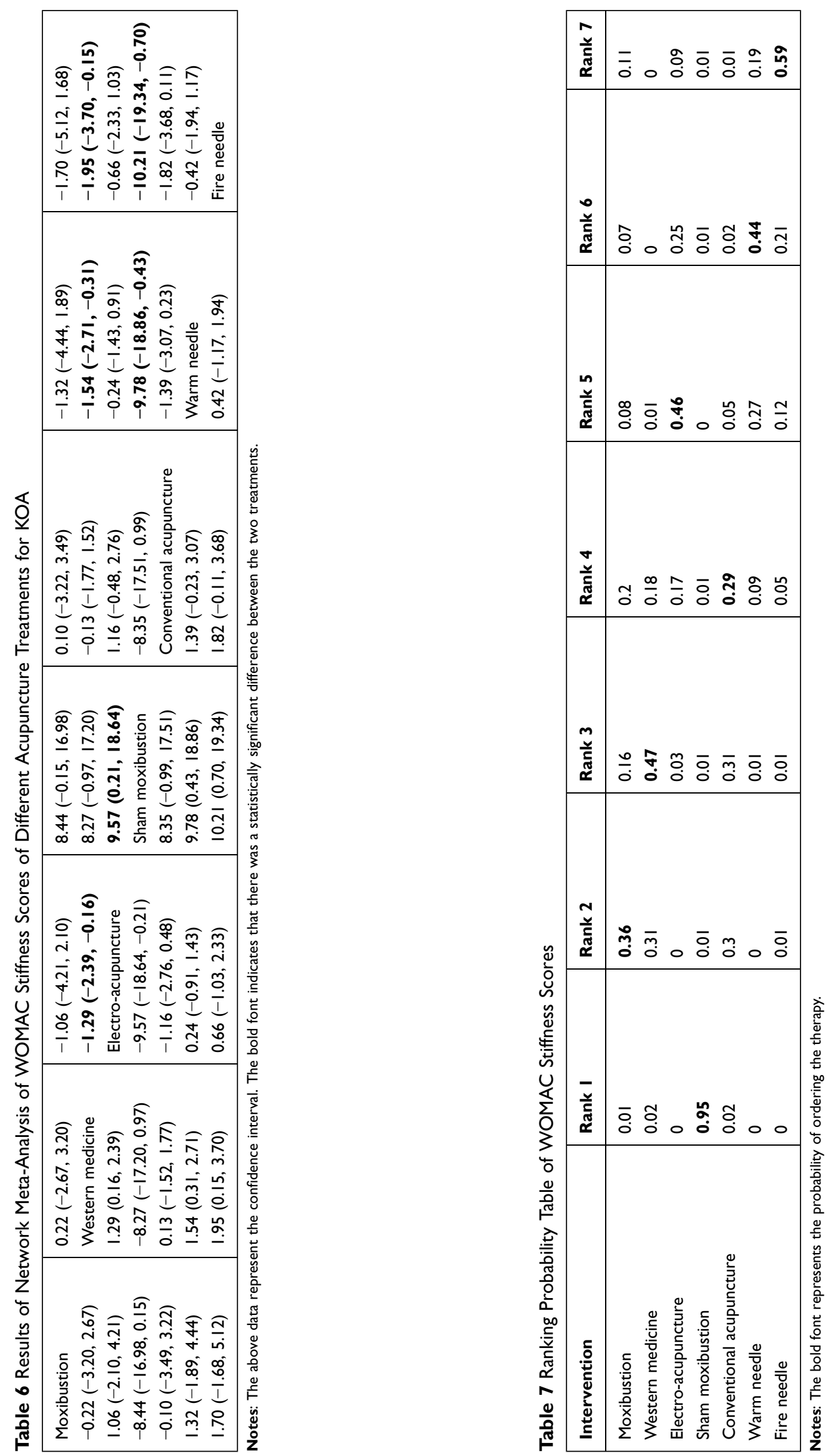

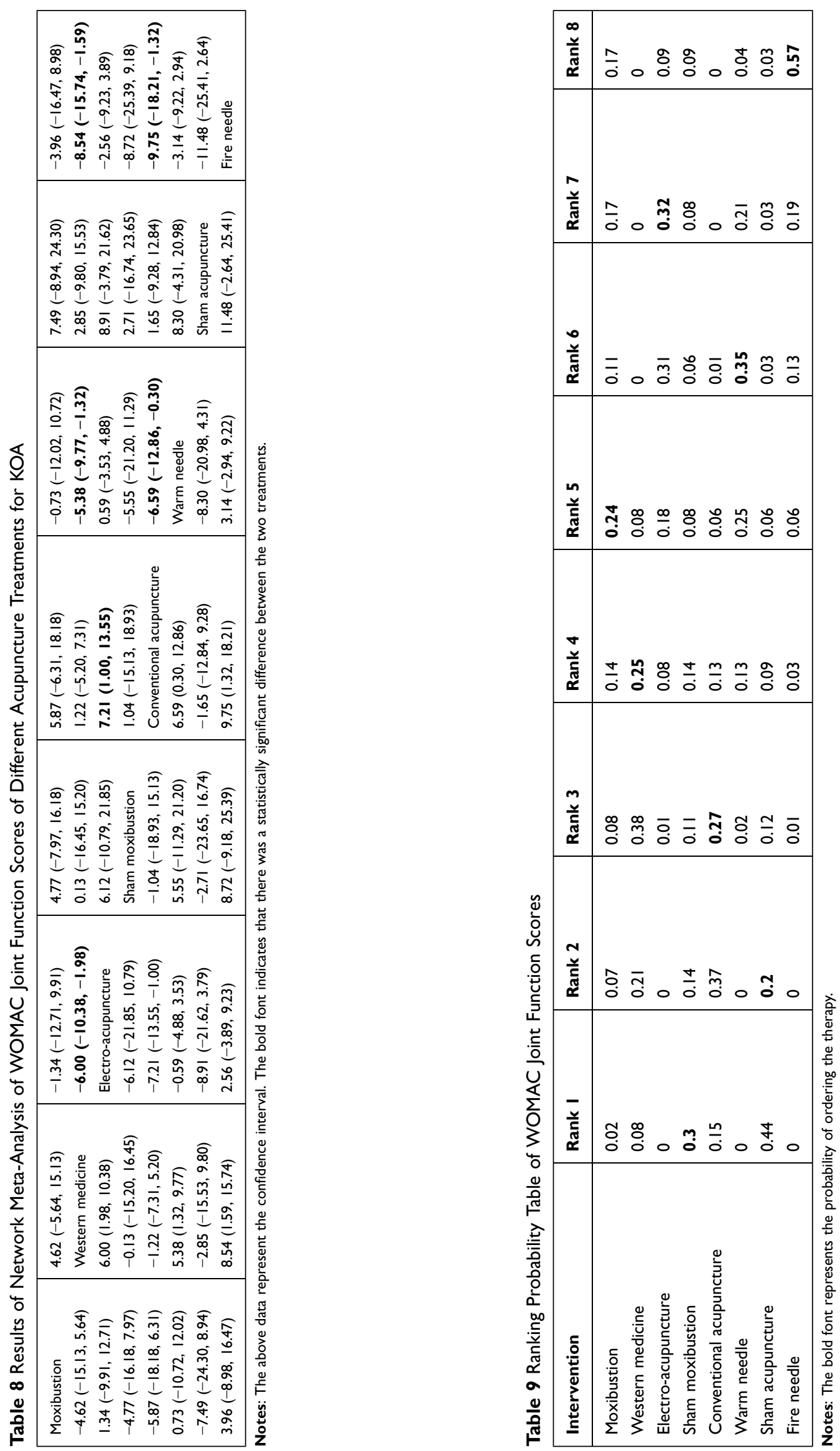


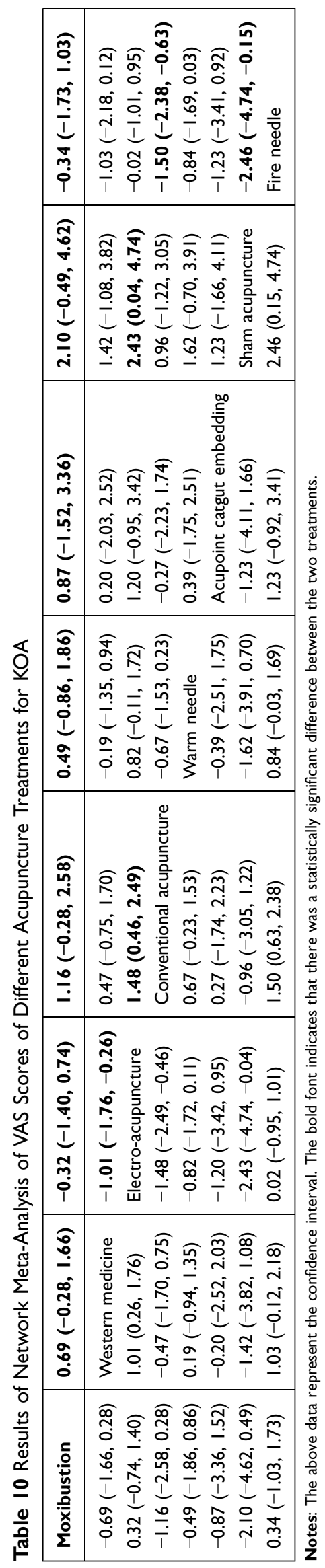

improving WOMAC pain scores. Probability ranking results in improving WOMAC pain scores showed that fire needle $>$ warm needle $>$ electro-acupuncture $>$ western medicine $>$ moxibustion $>$ conventional acupuncture $>$ sham acupuncture $>$ sham moxibustion. Moreover, electro-acupuncture was superior to western medicine and sham moxibustion while fire needle and warm needle were superior to western medicine and sham moxibustion in improving WOMAC stiffness scores. Probability ranking results in improving WOMAC stiffness scores showed that fire needle $>$ warm needle $>$ electro-acupuncture $>$ conventional acupuncture $>$ western medicine $>$ moxibustion $>$ sham moxibustion. Further analysis revealed that fire needle, warm needle and electro-acupuncture were all superior to conventional acupuncture and western medicine in improving WOMAC joint function scores. Probability ranking results in improving WOMAC joint function scores showed that fire needle $>$ electro-acupuncture $>$ warm needle $>$ moxibustion $>$ western medicine $>$ conventional acupuncture $>$ sham acupuncture $>$ sham moxibustion. Electro-acupuncture was more effectively improved VAS scores compared to western medicine, conventional acupuncture and sham acupuncture, while fire needle was superior to conventional acupuncture and sham acupuncture in improving VAS scores. Probability ranking results in improving VAS scores showed that fire needle $>$ electro-acupuncture $>$ moxibustion $>$ warm needle $>$ western medicine $>$ conventional acupuncture $>$ acupoint catgut embedding $>$ sham acupuncture. These results indicate that the fire needle had the best performance among the tested treatments in KOA treatment. Quality analysis results showed that the included studies had a medium quality. Thus, the application of the aforementioned interventions should be customized to the characteristics and condition of patients, and the probability ranking results only serve as a reference to clinicians.

Meta-analysis of previous online studies on the subject found that ${ }^{57}$ warm needle and electro-acupuncture were probably the best acupuncture modalities for treating KOA. In this study, different conclusions were drawn. Our results indicate that fire needle, electro-acupuncture, and warm needle ranked top of all tested therapies. The fire needle regulates IL-1 signal transduction pathways to balance articular cartilage synthesis and decomposition. In this way, it reduces inflammation and joint injury, promotes local blood circulation and alleviates clinical symptoms in patients. ${ }^{58,59}$ Electro-acupuncture therapy is also one of the most effective KOA treatments. ${ }^{60}$ Studies ${ }^{61,62}$ have reported that electro-acupuncture can reduce the 
Table I I Ranking Probability Table of VAS Scores

\begin{tabular}{|l|l|l|l|l|l|l|l|l|}
\hline Intervention & Rank I & Rank 2 & Rank 3 & Rank 4 & Rank 5 & Rank 6 & Rank 7 & Rank 8 \\
\hline Moxibustion & 0.01 & 0.02 & 0.04 & 0.09 & 0.19 & $\mathbf{0 . 3}$ & 0.19 & 0.17 \\
Western medicine & 0.04 & 0.14 & 0.22 & $\mathbf{0 . 3 2}$ & 0.23 & 0.05 & 0.01 & 0 \\
Electro-acupuncture & 0 & 0 & 0 & 0.01 & 0.05 & 0.2 & $\mathbf{0 . 4 2}$ & 0.33 \\
Conventional acupuncture & 0.09 & 0.46 & $\mathbf{0 . 3 4}$ & 0.09 & 0.03 & 0.01 & 0 & 0 \\
Warm needle & 0.01 & 0.04 & 0.15 & 0.33 & $\mathbf{0 . 3 1}$ & 0.14 & 0.03 & 0 \\
Acupoint Catgut embedding & 0.15 & $\mathbf{0 . 2 3}$ & 0.18 & 0.12 & 0.11 & 0.07 & 0.06 & 0.08 \\
Sham acupuncture & $\mathbf{0 . 7 I}$ & 0.13 & 0.06 & 0.04 & 0.03 & 0.02 & 0.01 & 0.01 \\
Fire needle & 0 & 0 & 0 & 0.01 & 0.07 & 0.22 & 0.29 & $\mathbf{0 . 4 1}$ \\
\hline
\end{tabular}

Notes: The bold font represents the probability of ordering the therapy.

expression of inflammatory cytokines in knee joints and inhibit inflammatory responses to achieve therapeutic effects. Warm needle suppresses inflammatory responses and alleviates clinical symptoms in KOA patients by inhibiting the expression of MMP-3 and TNF- $\alpha$ in joints. ${ }^{62}$ Warm needle up-regulates the expression of osteoprotegerin (OPG), down-regulates the expression of receptor activator of NF- $\mathrm{KB}$ Ligand (RANKL), and increases the ratio of $\mathrm{OPG} / \mathrm{RANKL}$, thereby reducing bone resorption in subchondral bone and inhibiting the destruction of subchondral bone in KOA. ${ }^{63}$

This study has some limitations: First, most of the included studies were not described in detail in the aspects of allocation concealment and blinding methods, and experimental designs were not rigorously evaluated which decreases the quality of results presented here. Second, sample sizes, type, dosage and treatment course of western medicine in the included literatures were not consistent, leading to potential heterogeneity. Third, the included studies had some publication bias and small sample effect, which decreases the reliability of our results.

In conclusion, this network meta-analysis show that the fire needle is superior to warm needle and electro-acupuncture, while warm needle and electro-acupuncture is better than conventional acupuncture, western medicine, sham moxibustion, sham acupuncture in overall curative effect. In clinical practice, appropriate treatments should be selected while considering the patient's situation. Due to the

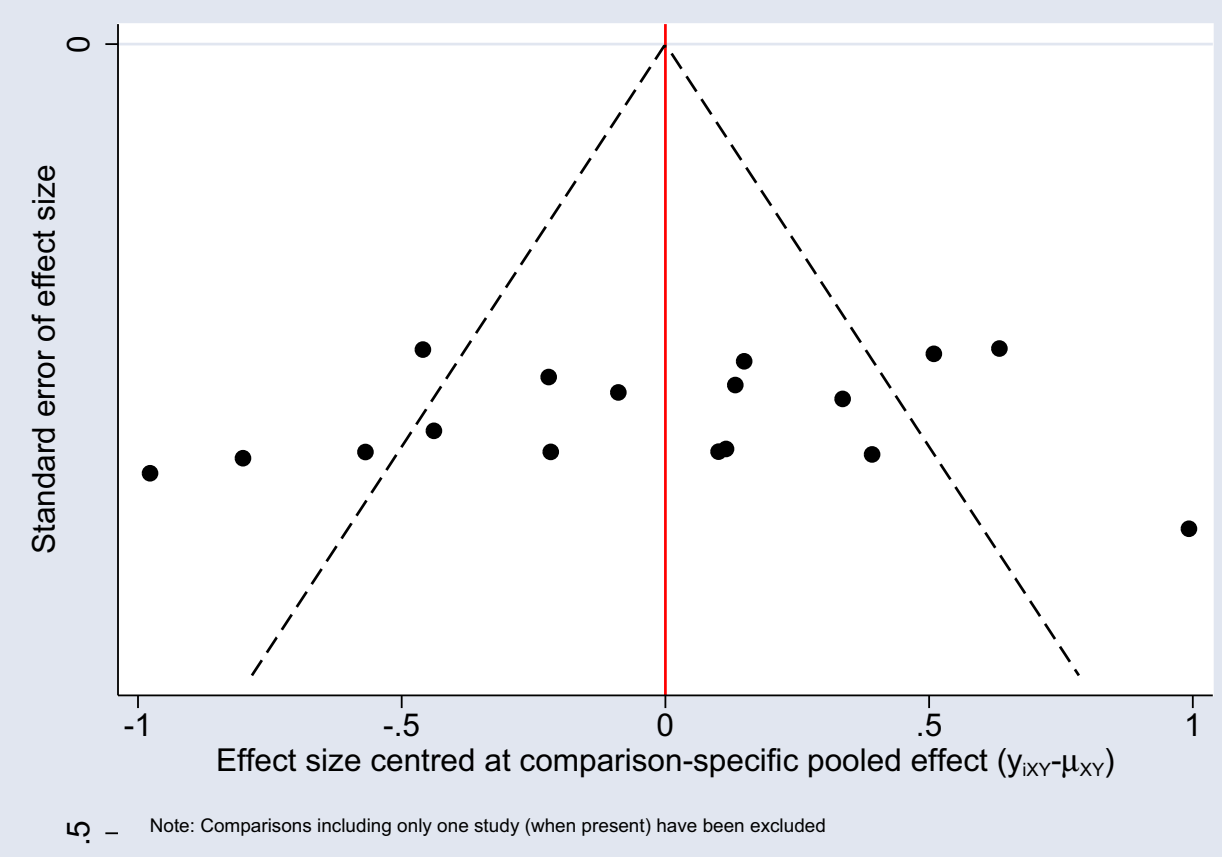

Figure 7 WOMAC pain score comparison corrected funnel plots of different acupuncture treatments for KOA. 
Table 12 Incidences of Adverse Events in Included Studies

\begin{tabular}{|c|c|}
\hline Included Studies & Adverse Events \\
\hline Zhang 2011"1 & $\begin{array}{l}\text { Moxibustion group: none } \\
\text { Western medicine group: } 3 \text { cases (sour regurgitation I, ausea I, epigastric pain I) }\end{array}$ \\
\hline Qiu $2006^{20}$ & $\begin{array}{l}\text { Electro-acupuncture group: } 2 \text { cases (local hematoma) } \\
\text { Western medicine group: } 9 \text { cases (epigastric discomfort, sour regurgitation) }\end{array}$ \\
\hline Yin $2017^{24}$ & $\begin{array}{l}\text { Electro-acupuncture group: I case (subcutaneous hemorrhage) } \\
\text { Western medicine group: } 2 \text { cases (gastrointestinal discomfort, mild constipation) }\end{array}$ \\
\hline Chen $2012^{31}$ & $\begin{array}{l}\text { Warm needle group: none } \\
\text { Western medicine group: I case(stomach discomfort) }\end{array}$ \\
\hline Jiang $2013^{32}$ & $\begin{array}{l}\text { Warm needle group: none } \\
\text { Western medicine group: none }\end{array}$ \\
\hline Shen $2017^{37}$ & $\begin{array}{l}\text { Conventional acupuncture group: } 2 \text { cases (fainting during acupuncture treatment) } \\
\text { Western medicine group: } 6 \text { cases (gastrointestinal discomfort, headache and dizziness, facial edema) }\end{array}$ \\
\hline Zhang $2016^{39}$ & $\begin{array}{l}\text { Warm needle group: } 2 \text { cases (fainting during acupuncture treatment, sticking of needle in acupuncture) } \\
\text { Conventional acupuncture group: } 8 \text { cases (constipation, diarrhea, nausea, dizziness) }\end{array}$ \\
\hline $\operatorname{Lin} 2018^{40}$ & $\begin{array}{l}\text { Conventional acupuncture group: } 2 \text { cases (pain and hematoma at the acupuncture site) } \\
\text { Sham acupuncture group: I case (pain at the acupuncture site) }\end{array}$ \\
\hline Zhao $2014^{41}$ & $\begin{array}{l}\text { Moxibustion group: } 10 \text { cases (reddening of skin) } \\
\text { sham moxibustion group: none }\end{array}$ \\
\hline Lu $201 I^{44}$ & $\begin{array}{l}\text { Fire needle group: none } \\
\text { Warm needle group: none }\end{array}$ \\
\hline $\operatorname{Lin} 2012^{48}$ & $\begin{array}{l}\text { Fire needle group: none } \\
\text { Conventional acupuncture group: none }\end{array}$ \\
\hline $\mathrm{He} 2018^{49}$ & $\begin{array}{l}\text { Electro-acupuncture group: none } \\
\text { Fire needle group: none }\end{array}$ \\
\hline
\end{tabular}

limitations associated with this study, future large scale, multi-center, high-quality randomized controlled trials are needed to validate results of this study.

\section{Abbreviations}

KOA, knee osteoarthritis; NSAIDs, Non-steroidal anti-inflammatory drugs; RCTs, Randomized controlled trials; WOMAC, Western Ontario and McMaster Universities Osteoarthritis Index; VAS, Visual Analogue Scale; MCMC, Markov Chain Monte Carlo; PSRF, potential scale reduction factor; ACR, American College of Rheumatology; TCM, traditional Chinese medicine; OARSI, Osteoarthritis Research Society International; OPG, osteoprotegerin; RANKL, receptor acti-

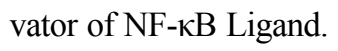

\section{Ethical Publication Statement}

We confirm that we have read the Journal's position on issues involving ethical publication and affirm that this report is consistent with those guidelines. Since this study is a literature analysis and does not involve patient privacy, informed consent of patients and approval of the ethics committee are not required.

\section{Acknowledgments}

The authors would like to thank Freescience (www.homefor-researchers.com) for English editing of this manuscript. Wei Liu, Yihua Fan and Yuanhao Wu have contributed equally to this work and are co-first authors.

\section{Funding}

This study was financially supported by the National Natural Science Foundation of China (No.82074377), the National Key Research and Development Project (No.2018YFC1705201), Tianjin Key Specialty Program (20210602-1), the Special Scientific Research for Traditional Chinese Medicine (No.201507001-07), and 
High-Level Talent Selection and Training Project of Tianjin Health and Family Planning Industry (02005wuyuanhao).

\section{Disclosure}

The authors report no conflicts of interest in this work.

\section{References}

1. Dawson J, Linsell L, Zondervan K, et al. Impact of persistent hip or knee pain on overall health status in elderly people: a longitudinal population study. Arthritis Rheum. 2005;53(3):368-374. doi:10.1002/ art. 21180

2. Palmer KT, Reading I, Calnan M, Linaker C, Coggon D, Palmer KT, Reading I, Calnan M, et al. Does knee pain in the community behave like a regional pain syndrome? Prospective cohort study of incidence and persistence. Ann Rheum Dis. 2007;66(9):1190-1194. doi:10.1136/ard.2006.061481

3. Avendaño-Coy J, Comino-Suárez N, Grande-Muñoz J, et al. Extracorporeal shockwave therapy improves pain and function in subjects with knee osteoarthritis: a systematic review and meta-analysis of randomized clinical trials. Int J Surg. 2020;82:64-75. doi:10.1016/j.ijsu.2020.07.055

4. Lu YH, Shi XB. Current status and progress of epidemiological studies on knee osteoarthritis. Chine $J$ Traditional Med Traumatology Orthopedics. 2012;20(6):81-84.

5. Blagojevic M, Jinks C, Jeffery A, et al. Risk factors for onset of osteoarthritis of the knee in older adults: a systematic review and meta-analysis. Osteoarthritis Cartilage. 2010;18(1):24-33. doi:10.1016/j.joca.2009.08.010

6. McAlindon TE, Bannuru RR, Sullivan MC, et al. OARSI guidelines for the non-surgical management of knee osteoarthritis. Osteoarthritis Cartilage. 2014;22(3):363-388. doi:10.1016/j. joca.2014.01.003

7. Comino-Suárez N. Guidelines for Diagnosis and Treatment of Osteoarthritis. Chine J Rheumatol. 2010;14(6):416-419.

8. Berman BM, Lao L, Langenberg P, Lee WL, Gilpin AMK, Hochberg MC, Berman BM, Lao L, Langenberg P, et al. Effectiveness of acupuncture as adjunctive therapy in osteoarthritis of the knee: a randomized, controlled trial. Ann Intern Med. 2004;141(12):901910. doi:10.7326/0003-4819-141-12-200412210-00006

9. Tukmachi E, Jubb R, Dempsey E, et al. The effect of acupuncture on the symptoms of knee osteoarthritis-an open randomised controlled study. Acupunct Med. 2004;22(1):14-22. doi:10.1136/aim.22.1.14

10. McGrory B, Weber K, Lynott JA, et al. American Academy of Orthopaedic Surgeons. The American Academy of Orthopaedic Surgeons Evidence-Based Clinical Practice Guideline on Surgical Management of Osteoarthritis of the Knee. J Bone Joint Surg Am. 2016;98(8):688-692. doi:10.2106/JBJS.15.01311

11. Zhang QJ, Cao LH, Li ZD, et al. Clinical effect and safety observation of moxibustion and celecoxib in the treatment of knee osteoarthritis. Chine J Traditional Med Traumatology Orthopedics. 2011;19 (1):13-15.

12. Zhou YL, Li J, Hou WG, et al. Clinical observation on moxibustion treatment of knee osteoarthritis. Shanghai $J$ Acupuncture Moxibustion. 2014;33(12):1086-1088.

13. Zhou JY. Double blind and double simulated clinical randomized controlled study of moxibustion treatment of knee osteoarthritis. $J$ Chengdu Univ Traditional Chin Med. 2017.

14. Ren XM, Cao JJ, Shen XY, et al. Moxibustion for knee osteoarthritis: a randomized controlled study. Chin Acupuncture Moxibustion. 2011;31(12):1057-1061.
15. Zhou T, Chen CF, Qian YL, et al. Clinical Effect of Low frequency electro-acupuncture on knee osteoarthritis. Shanghai J Traditional Chine Med. 2015;49(8):56-57.

16. Zhang H, Lv ZT, Lei C, et al. Clinical Efficacy of electro-acupuncture on Knee Osteoarthritis and Effect of Serum GRO- $\alpha$ and VEGF. $J$ Clin Acupuncture Moxibustion. 2018;34(10):22-24.

17. Liu N, Cai CM, Fu YT, et al. Clinical Observation on 41 Cases of Knee Osteoarthritis Treated by electro-acupuncture and Excision. World J Integrated Traditional Western Med. 2020;15 (2):344-347.

18. Guo YM, Liang YY, Gu JQ, et al. Electro-acupuncture around acupuncture treatment of early and middle knee osteoarthritis. Jilin $J$ Chine Med. 2016;36(1):91.

19. Wu WH, Tang J, Wu YP, et al. Evaluation of modern rehabilitation of the curative effect of electro-acupuncture on Xiyan(EX-LE5) point in the treatment of knee osteoarthritis. Shanghai J Traditional Chine Med. 2015;49(06):63-65.

20. Qiu ML, Dai QP, Che T, et al. Clinical study of electro-acupuncture at knee eye point in the treatment of knee osteoarthritis. $J$ Traditional Chine Orthopedics Traumatol. 2006;18(3):15-16.

21. Gang JH, Mi YQ, Wang HM. Clinical efficacy of electro-acupuncture versus meloxicam in the treatment of early -and mid-stage knee osteoarthritis: a randomized controlled study. Chin Acupuncture Moxibustion. 2016;36(05):467-470.

22. Gao J, Ouyang BS, Zhang Y, et al. Comparison of curative effect between electro-acupuncture and warming acupuncture moxibustion on knee osteoarthritis of kidney deficiency and myeloid deficiency. Chin Acupuncture Moxibustion. 2012;32(5):395-398.

23. Wang J. electro-acupuncture and warm needle in the treatment of blood stasis type knee joint osteoarthritis. Lab Med Clini. 2017;14(4):508-509.

24. Yin XZ. Effect of electro-acupuncture on functional improvement of knee osteoarthritis. Chine General Pract. 2017;20(S3):388-389.

25. Ju ZY, Wang Y, Chen CL, et al. Clinical study of electro-acupuncture on knee osteoarthritis. Shanghai J Acupuncture Moxibustion. 2017;36 (09):1111-1115.

26. Wu JL. Clinical observation of electro-acupuncture on knee osteoarthritis. J Heilongjiang Univ Traditional Chin Med. 2012.

27. Chen ZX. Effect of warm needle on pain and quality of life of senile knee osteoarthritis. Clin J Chine Med. 2018;10(5):86-87+89.

28. Liu RB, Huang $\mathrm{Z}$, Wu Q. Clinical Observation of Warming Acupuncture and moxibustion on Knee Osteoarthritis of qi-stagnation and blood stasis type. J Guangzhou Univ Traditional Chine Med. 2014;9(1). doi:10.1186/1749-8546-9-2

29. Tan HY, Feng YL. Clinical Effect of Warming Acupuncture and Acupuncture on the Dubi(ST35) and Neixiyan(EX-LE4) in the Treatment of Knee Osteoarthritis. Clin J Chine Med. 2016;8(14):23-24.

30. Ma SJ, Yang K, Li FQ. Clinical observation on treating 54 cases of knee osteoarthritis with warm needle. Zhejiang $J$ Traditional Chine Med. 2015;50(10):751.

31. Chen ZG, Wu LH, Chen MJ, et al. Long-term effect of warm needle on knee osteoarthritis. Shanghai J Acupuncture Moxibustion. 2012;31 (5):339-341.

32. Jiang B, Hou XQ, Tang ZY. Clinical study on warm needle treatment of knee osteoarthritis. Chine J Traditional Med Traumatology Orthopedics. 2013;21(11):18-21.

33. Ren ZJ. Clinical study on warm needle treatment of knee osteoarthritis. China Health Standard Management. 2018;9(8):94-96.

34. Zhang J. Therapeutic effect of warm needle on knee osteoarthritis of deficiency and cold type. China Health Standard Management. 2016;7(12):128-129.

35. Pan YY, Tang XJ, Cui WQ, et al. Clinical observation of acupoint catgut embedding in the treatment of knee osteoarthritis. Chine Med Modern Distance Educ China. 2020;18(09):90-92.

36. Ji LL. Clinical study on the effect of acupuncture and moxibustion on serum cytokines in patients with knee osteoarthritis. J Nanjing Univ Traditional Chine Med. 2012. 
37. Shen J. A comparative study of acupuncture and moxibustion therapy and diclofenac sodium emulsion in the treatment of senile knee osteoarthritis. J Clin Acupuncture Moxibustion. 2017;33(7):22-25.

38. Zhang SL, He YY, He SM. Clinical Observation of 28 Cases of Knee Osteoarthritis Treated by Acupuncture. J Med Theory Pract. 2016;29 (22):3084-3085.

39. Miao GD, Jiang ZF, Tian ZJ. Observation on the curative effect of acupuncture and moxibustion on knee osteoarthritis. Hebei J Traditional Chine Med. 2014;36(5):725-726.

40. Lin LL, Li YT, Tu JF, et al. Effectiveness and feasibility of acupuncture for knee osteoarthritis: a pilot randomized controlled trial. Clin Rehabil. 2018;32(12):1666-1675. doi:10.1177/0269215518790632

41. Zhao L, Cheng K, Wang L, et al. Effectiveness of moxibustion treatment as adjunctive therapy in osteoarthritis of the knee: a randomized, double-blinded, placebo-controlled clinical trial. Arthritis Res Ther. 2014;16(3):R133. doi:10.1186/ar4590

42. Zhang GB, Wang WL, Zhang J, et al. A comparative study of fire needle and warm needle in the treatment of knee osteoarthritis. Shanghai J Acupuncture Moxibustion. 2013;32(4):299-301.

43. Zheng M, Hong KD, Chen SJ, et al. Observation on the curative effect of fire needle on knee osteoarthritis. Chine Med Modern Distance Educ China. 2016;14(5):103-106.

44. Lu DJ, Wang Q, Mei SW. Clinical comparative study of fire needle and warm needle in the treatment of knee osteoarthritis. J New Chine Med. 2011;43(10):94-96.

45. Li T, Ren J, Li LY. Study on the curative effect of fire needle on early and middle stage of knee osteoarthritis. J Xinjiang Med Univ. 2020;43(5):629-633.

46. Tang LT. Comparison and observation on the clinical effect of fire needle and warm needle therapy in treating knee osteoarthritis of yang deficiency and cold coagulation type. J Guangxi Univ Chine Med. 2017.

47. Song WP. Clinical Observation on the Treatment of Knee Osteoarthritis of Yang-deficiency and Cold Coagulation type whit fire needle. Yunnan J Traditional Chine Med Materia Med. 2020;41 (6):64-66.

48. Lin JC. Clinical Observation on the Curative Effect of Fire Needle on Knee Osteoarthritis. Jiangsu: Nanjing University of Traditional Chinese Medicine; 2011.

49. He TF, Bing XH, Zhou LY, et al. Comparison of curative effect of fire needle and electro-acupuncture on knee osteoarthritis. Shanghai J Acupuncture Moxibustion. 2018;37(6):675-679.

50. Fan CL, Zhang Q, Xu JH, et al. Clinical Observation of 54 Cases of Knee Osteoarthritis of Yang Deficiency and Cold Congealment type treated by fire needle. World J Integrated Traditional Western Med. 2016;11(4):524-526+530.

51. Bannuru RR, Osani MC, Vaysbrot EE, et al. OARSI guidelines for the non-surgical management of knee, hip, and polyarticular osteoarthritis. Osteoarthritis Cartilage. 2019;27(11):1578-1589. doi:10.1016/j.joca.2019.06.011
52. Hepper CT, Halvorson JJ, Duncan ST, et al. The efficacy and duration of intra-articular corticosteroid injection for knee osteoarthritis: a systematic review of level I studies. $J$ Am Acad Orthop Surg. 2009;17(10):638-646. doi:10.5435/00124635-200910000-00006

53. Han Y, Huang H, Pan J, et al. Meta-analysis Comparing Platelet-Rich Plasma vs Hyaluronic Acid Injection in Patients with Knee Osteoarthritis. Pain Med. 2019;20(7):1418-1429. doi:10.1093/pm/ pnz011

54. Li J, Li YX, Luo LJ, et al. The effectiveness and safety of acupuncture for knee osteoarthritis: an overview of systematic reviews. Medicine. 2019;98(28):e16301.

55. Li-Jiang J, Jing-Yu X, Yang X-J, et al. A study of acupoint selection rules of Moxibustion and Auricular point therapy in the treatment of urinary retention after anorectal surgery based on data mining. Asian Toxicol Res. 2021;3(1):4.

56. Corbett MS, Rice SJ, Madurasinghe V, et al. Acupuncture and other physical treatments for the relief of pain due to osteoarthritis of the knee: network meta-analysis. Osteoarthritis Cartilage. 2013;21 (9):1290-1298. doi:10.1016/j.joca.2013.05.007

57. Li S, Xie P, Liang Z, et al. Efficacy comparison of five different acupuncture methods on pain, stiffness, and function in osteoarthritis of the knee: a network meta-analysis. J Ningxia Med Univ. 2018;2018:1638904.

58. Wang WJ, Geng P, Chen DS, et al. Effects of fire needle on IL-1 Signal Transduction Pathway of Knee Osteoarthritis. Pract Clin J Integrated Traditional Chin Western Med. 2015;15(5):29-30+41.

59. Wang Y, Xie X, Zhu X, et al. Fire-Needle Moxibustion for the Treatment of Knee Osteoarthritis: a Meta-Analysis. Evid Based Complementary Alternative Med. 2016;2016:1392627.

60. Kang HR, Lee YS, Kim SH, et al. Effectiveness and safety of electrical moxibustion for knee osteoarthritis: a multicenter, randomized, assessor-blinded, parallel-group clinical trial. Complement Ther Med. 2020;53:102523. doi:10.1016/j.ctim.2020.102523

61. Kang HR, Jung CY, Lee SD, et al. Efficacy and safety of electrical moxibustion for knee osteoarthritis: study protocol for a randomized controlled trial. Trials. 2018;19(1):159. doi:10.1186/s13063-0182514-X

62. Yue P, Gao L, Chen M, et al. Effect of Warm-needle-moxibustion on Behavior Reactions and TNF- $\alpha$ and MMP-3 Contents in Knee Cartilage of Rabbits with Knee Osteoarthritis. Acupuncture Res. 2016;41(3):235-239.

63. Ma YY, Liu D, Liu JW, et al. Effect of warm needle on expression of OPG and RANKL protein in subchondral bone of knee osteoarthritis patients. J Ningxia Med Univ. 2020;42(7):683-687.
Journal of Pain Research

\section{Publish your work in this journal}

The Journal of Pain Research is an international, peer reviewed, open access, online journal that welcomes laboratory and clinical findings in the fields of pain research and the prevention and management of pain. Original research, reviews, symposium reports, hypothesis formation and commentaries are all considered for publication. The manuscript management system is completely online and includes a very quick and fair peer-review system, which is all easy to use. Visit http:// www.dovepress.com/testimonials.php to read real quotes from published authors. 